Y. Kato

Nagoya Math. J.

Vol. 86 (1982), 1-38

\title{
ON NON-ELLIPTIC BOUNDARY PROBLEMS
}

\author{
YOSHIO KATO
}

\section{Introduction}

The purpose of this paper is to study the boundary value problems for the second order elliptic differential equation

$$
A U=-\sum_{i, j=1}^{n} \partial_{i}\left(a_{i j} \partial_{j} U\right)+\sum_{i=1}^{n} b_{i} \partial_{i} U+c U=F
$$

in a bounded domain $\Omega$ in $R^{n}(n \geqq 3)$ with the boundary condition

$$
B U=\sum_{i=1}^{n} \alpha_{i} \partial_{i} U+\beta U=f
$$

on the boundary $\Gamma$ of $\Omega$, where we assume that

1) for every $x \in \Gamma$, the inequality

$$
\sum_{i=1}^{n} \alpha_{i}(x)^{2}>0
$$

holds,

2) let $\left(n_{1}(x), \cdots, n_{n}(x)\right)$ be the exterior unit normal vector to $\Gamma$ at $x$, then the subset of $\Gamma$,

$$
\Gamma_{0}=\left\{x \in \Gamma ; \sum_{i=1}^{n} \alpha_{i}(x) n_{i}(x)=0\right\}
$$

is a $C^{\infty}$-manifold of dimension $n-2$,

$3)$ at every point $x \in \Gamma_{0}$, the $n$-vector $\left(\alpha_{1}(x), \cdots, \alpha_{n}(x)\right)$ is not tangent to $\Gamma_{0}$.

Here $\partial_{i}$ denotes $\partial / \partial x_{i}, a_{i j}$ is symmetric on $\Omega$, and $\Gamma$ is assumed to be infinitely smooth and of dimension $n-1$. We further assume that the coefficients of the equations (1) and (2) are real-valued and infinitely differentiable on $\bar{\Omega}=\Omega \cup \Gamma$ and $\Gamma$, respectively, and that there exists a positive constant $c_{0}$ such that

Received February 21, 1977. 


$$
\sum a_{i j}(x) \xi_{i} \xi_{j} \geqq c_{0}|\xi|^{2}
$$

holds for all $x \in \bar{\Omega}$ and $\xi \in R^{n}$.

This problem was investigated by Maljutov [6] on probability considerations, by Egorov-Kondrat'ev [1] while they are developing some ideas of Hörmander [2], by Soga [7] and others. In the present paper we shall try to solve the problem (1)-(2) by using the similar argument as in [5].

If we set

$$
\alpha^{\prime}(x)=\sum_{i=1}^{n} \alpha_{i}(x) n_{i}(x),
$$

the boundary condition (2) can be written in the form

$$
B U=\alpha^{\prime} \frac{\partial U}{\partial n}+\gamma^{\prime} U+\beta U=f
$$

with the suitable tangential vector field $\gamma^{\prime}$. Using the conormal vector field $\nu=\left(\nu_{1}, \cdots, \nu_{n}\right)$ with

$$
\nu_{j}(x)=\sum_{i=1}^{n} a_{i j}(x) n_{i}(x)
$$

and setting

$$
\frac{\partial U}{\partial n}=a_{0}(x) \frac{\partial U}{\partial \nu}+\gamma_{0} U \quad\left(\frac{\partial U}{\partial \nu}=\sum_{j=1}^{n} \nu_{j} \frac{\partial U}{\partial x_{j}}\right),
$$

we can rewrite $\left(2^{\prime}\right)$ as

$$
B U=\alpha \frac{\partial U}{\partial \nu}+\gamma U+\beta U=f
$$

with $\alpha=\alpha^{\prime} a_{0}$ and $\gamma=\alpha^{\prime} \gamma_{0}+\gamma^{\prime}$, where $a_{0}(x)$ is a positive $C^{\infty}$-function on $\Gamma$ and $\gamma_{0}$ is also a tangential vector field. Assumptions 1), 2) and 3) yield that $\alpha(x)$ vanishes only on $\Gamma_{0}$, that $\gamma$ is transversal to $\Gamma_{0}$, and that the boundary condition ( $\left.2^{\prime \prime}\right)$ is elliptic (i.e. satisfies the Lopatinsky condition) on $\Gamma$ except for $\Gamma_{0}$, that is, $\Gamma_{0}$ is a singular manifold for the boundary value problem (1)-(2). For the sake of simplicity, we assume that $\Gamma_{0}$ is connected. Following Egorov-Kondrat'ev we can then classify the singular manifold $\Gamma_{0}$, by denoting $\Gamma_{+}\left(\Gamma_{-}\right)=\{x \in \Gamma ; \alpha(x)>0(\alpha(x)<0)\}$, as follows; 
(I) $\Gamma_{-}=\phi$ (i.e. $\alpha \geqq 0$ throughout $\Gamma$ ) or

$\Gamma_{+}=\phi$ (i.e. $\alpha \leqq 0$ throughout $\Gamma$ ),

(II) $\Gamma_{+} \neq \phi, \Gamma_{-} \neq \phi$ and $\gamma$ is transversal from $\Gamma_{-}$to $\Gamma_{+}$on $\Gamma_{0}$,

(III) $\Gamma_{+} \neq \phi, \Gamma_{-} \neq \phi$ and $\gamma$ is transversal from $\Gamma_{+}$to $\Gamma_{-}$on $\Gamma_{0}$.

It is clear that $\Gamma_{0}$ is a closed manifold in case (II) or (III).

In Chapter 1, we reduce the boundary problem (1)-(2") to the pseudo-differential equation $(\alpha S+\gamma+\beta) u=f$ on $\Gamma$ (Proposition 1.2) and introduce Hilbert spaces in which solutions of the equation are seeked by making use of the Lax-Milgram theorem. In Chapters 2, 3 and 4, we consider the boundary conditions, according to cases (I), (II) and (III), respectively. As in [5], we use the variational approach, and apply the elliptic regularization. A special feature of the proofs is to introduce the appropriate auxiliary functions $h$ in the respective types (I), (II) and (III) so that $h \alpha$ is positive on $\Gamma \backslash \Gamma_{0}$ and vanishes on $\Gamma_{0}$, etc. (see Lemmas 2.1 and 3.1), and to consider the equations $P u=f$ instead of the equation $(\alpha S+\gamma+\beta) u=f$, where $P=h(\alpha S+\gamma+\beta)$ in case (I), $P=(\alpha S+\gamma+\beta) h$ in case (II) and $P=h(\alpha S+\gamma+\beta)$ in case (III). Fortunately, we can choose in respective cases pseudo-differential operators $H$ of order zero so that $(P+H) u=f$ are uniquely solvable for all $f$ in some functional spaces. If we set $u=K f$, it can be proved that the equation $P u=f$ is altered to the equation $(1-H K) g=f$ with $u=K g$. In order to solve the latter equation, it is sufficient to show that the operator $H K$ is compact (to apply the Riesz-Schauder theory).

In $\S \S 2.1,3.1$ and 4.1, we introduce $h, H$ and treat $(P+H) u=f$. The equation $P u=f$ is considered in $\S \S 2.2,3.2$ and 4.2. Sections 2.3, 3.3 and 4.3 are devoted to the uniqueness of solutions of $P u=f$. Finally, in $\S \S 2.4,3.4$ and 4.4 , we return to the original problem (1)-(2) and prove the uniqueness, the existence and the regularity.

For the more general case where the singular manifold $\Gamma_{0}$ consists of finite number of disjoint manifold of types (I), (II) and (III), we can also formulate the similar results by virtue of the results obtained in the respective types and their local character.

Recently, in [8] Winzell investigates the problem (1)-(2) $(\beta=0)$, allowing $\Gamma_{0}$ to be fairly complicated and to have a certain width.

\section{Chapter 1. Preliminaries}

1.0. Let $\Omega$ be a bounded domain of $R^{n}$ with $C^{\infty}$-boundary $\Gamma$ of dimen- 
sion $n-1$ and let $A$ be the second order elliptic differential operator described in Introduction. Here we assume that $\Omega$ is of the form $\Gamma \times(0,1)$ near $\Gamma$ and that $A$ is defined and is elliptic in a larger domain $\Omega_{1}$ with $C^{\infty}$-boundary such that $\bar{\Omega} \subset \Omega_{1}$. Near $\Gamma$ we choose a coordinate system $\left(x^{\prime}, x_{n}\right)$ such that $x^{\prime} \in \Gamma$ and $x_{n}$ is a normal coordinate on $x^{\prime}$. Let $\left\{\omega_{i}\right\}_{i=1}^{\ell}$ be a finite open covering of $\Gamma$ and $\kappa_{i}$ be a $C^{\infty}$-coordinate transformation $y=\kappa_{i}(x)$ such that $\omega_{i}$ is mapped onto an open ball $B_{i}$ in $R_{y}^{n-1}$ with the origin as center and $\omega_{i} \cap \Gamma_{0}$ onto $\left\{y_{1}=0\right\} \cap B_{i}$ if $\omega_{i} \cap \Gamma_{0} \neq \phi$, and such that $\gamma$ is transformed to $\partial / \partial y_{1}$ on $\omega_{i}$ such that $\omega_{i} \cap \Gamma_{0} \neq \phi$. Let $\left\{\zeta_{i}\right\}_{i=1}^{\ell}$ be a partition of unity subordinate to the covering $\left\{\omega_{i}\right\}_{i=1}^{\ell}$ such that $\gamma\left(\zeta_{j}\right)=0$ in a neighborhood of $\Gamma_{0}$ for all $j$.

Let $E_{s}$ ( $s$ : real) be a pseudo-differential operator on $R^{n-1}$ defined by

$$
\left(E_{s} u\right)(y)=(2 \pi)^{1-n} \int_{R^{n-1}}\left(1+|\xi|^{2}\right)^{s / 2} \hat{u}(\xi) e^{i y \xi} d \xi
$$

where

$$
\hat{u}(\xi)=\int_{R^{n-1}} u(y) e^{-i y \xi} d y .
$$

Now $H_{s}(\Gamma)$ is the usual Sobolev space with the norm

$$
\left\{\begin{array}{l}
\|u\|_{s}=\left(\sum_{j=1}^{\ell} \int\left|E_{s}\left(\tilde{\zeta}_{j} \tilde{u}\right)\right|^{2} d y\right)^{1 / 2} \quad(s \neq 0) \\
\|u\|_{0}=\int_{\Gamma}|u|^{2} d \sigma
\end{array}\right.
$$

where, as well as in the below, $\tilde{v}(y)$ denotes a function on $B_{j}$ defined by

$$
\tilde{v}(y)=v\left(\kappa_{j}^{-1}(y)\right), \quad v \in \mathscr{D}^{\prime}\left(\omega_{j}\right)
$$

and $d \sigma$ is the Lebesgue measure on $\Gamma$.

1.1. Following [2], in a neighborhood of $\Gamma$ we write the differential operator $A$ in (1) in the form $A=\sum_{j=0}^{2} A_{j} D_{n}^{j}$, where $D_{n}=i^{-1} \partial / \partial x_{n}(i=$ $\sqrt{-1}$ ) and $A_{j}$ is a differential operator of order $2-j$ acting along the parallel surface of $\Gamma$. Throughout this paper, we suppose the existence of the Green kernel $G$ (pseudo-differential operator on $\Omega_{1}$ ) of $A$ for the Dirichlet problem on $\Omega_{1}$. We denote by $\delta$ the surface measure on $\Gamma$. Then according to [2, Sections 2.1 and 2.2], we can prove the following

Proposition 1.1. (a) Let $U$ be in $H_{s}(\Omega)$ with real $s$ so that $A U=0$ in $\Omega$. Then the restriction of $D_{n}^{j} U$ on $\Gamma, u_{j}=\left.D_{n}^{j} U\right|_{\Gamma}$, is well defined in 
$H_{s-j-1 / 2}(\Gamma)$ for $j=0,1$, and $U$ is written in the form

$$
U=i^{-1} \sum_{j=0}^{1} \sum_{k=0}^{1-j} G A_{j+k+1} D_{n}^{j}\left(u_{k} \delta\right) .
$$

Furthermore, $u_{0}$ and $u_{1}$ satisfy

$$
u_{0}=Q_{0} u_{0}+Q_{1} u_{1},
$$

where $Q_{k}(k=0,1)$ are the pseudo-differential operators on $\Gamma$ of order $-k$ defined by

$$
Q_{k} v=\left.i^{-1} \sum_{j=0}^{1-k} G A_{j+k+1} D_{n}^{j}(v \delta)\right|_{\Gamma} \quad(k=0,1)
$$

(b) Let $u_{j}(j=0,1)$ be in $H_{s-j-1 / 2}(\Gamma)$ (s real). If $u_{0}$ and $u_{1}$ satisfy (1.3), then the $U$ given by (1.2) is a distribution in $H_{s}(\Omega)$ such that $A U=0$ in $\Omega, u_{0}=\left.U\right|_{\Gamma}$ and $u_{1}=\left.D_{n} U\right|_{\Gamma}$. Moreover there exists a constant $c>0$ such that

$$
c^{-1}\|U\|_{s, \Omega} \leqq\left\|u_{0}\right\|_{s-1 / 2}+\left\|u_{1}\right\|_{s-3 / 2} \leqq c\|U\|_{s, \Omega},
$$

where $\|\cdot\|_{s, \Omega}$ represents the norm in the usual Sobolev space $H_{s}(\Omega)$.

(c) The $Q_{1}$ defined by (1.4) is actually elliptic and invertible, and the operator

$$
S_{0}=i^{-1} Q_{1}^{-1}\left(1-Q_{0}\right)
$$

is a pseudo-differential operator on $\Gamma$ of order 1 . Moreover there exist two constants $c_{1}^{\prime}>0$ and $c^{\prime}$ such that the inequality

$$
\operatorname{Re}\left(S_{0} \phi, \phi\right) \geqq c_{1}^{\prime}\|\phi\|_{1 / 2}^{2}-c^{\prime}\|\phi\|_{0}^{2}
$$

holds for every $\phi \in C^{\infty}(\Gamma)$, where

$$
(u, v)=\int_{\Gamma} u \bar{v} d \sigma
$$

Proof. We refer to [2] for the proof of (a) and (b). Let $\pi$ be a pseudo-differential operator given by

$$
\pi(\phi)=\left.G(\phi \delta)\right|_{\Gamma}, \quad \phi \in C^{\infty}(\Gamma) .
$$

Then we can write

$$
\operatorname{Re}(\pi \phi, \phi)=\operatorname{Re} \int_{\Omega_{1}} G(\phi \delta) \cdot \overline{\phi \delta} d x
$$


noting that $\phi \delta \in H_{-1}\left(\Omega_{1}\right)$ and $G(\phi \delta) \in H_{1}\left(\Omega_{1}\right)$. On the other hand, for any $f \in C^{\infty}\left(\bar{\Omega}_{1}\right)$, we have setting $u=G f$

$$
\operatorname{Re} \int_{\Omega_{1}} G f \cdot \bar{f} d x=\operatorname{Re} \int_{\Omega_{1}} u \cdot A \bar{u} d x \geqq c\|u\|_{1, \Omega_{1}}^{2} \geqq c^{\prime}\|f\|_{-1, \Omega_{1}}^{2},
$$

with suitable constants $c>0, c^{\prime}>0$. Let $\left\{f_{n}\right\}_{n=1}^{\infty}$ be a sequence in $C^{\infty}\left(\bar{\Omega}_{1}\right)$ such that $f_{n}$ converges to $\phi \delta$ in $H_{-1}\left(\Omega_{1}\right)$ as $n \rightarrow \infty$. Applying (1.7) to $f=f_{n}$ and letting $n$ to infinity, we obtain

$$
\operatorname{Re} \int_{\Omega_{1}} G(\phi \delta) \cdot \overline{\phi \delta} d x \geqq c^{\prime}\|\phi \delta\|_{-1, \Omega_{1}}^{2} \geqq c^{\prime \prime}\|\phi\|_{-1 / 2}^{2},
$$

with a constant $c^{\prime \prime}>0$ independent of $\phi$. It then follows from (1.6) that the inequality

$$
\operatorname{Re}(\pi \phi, \phi) \geqq c^{\prime \prime}\|\phi\|_{-1 / 2}^{2}
$$

holds for every $\phi \in C^{\infty}(\Gamma)$. This shows that $\pi$ and hence $Q_{1}=i^{-1} \pi A_{2}$ are elliptic of order -1 and invertible. Therefore the operator $S_{0}=$ $i^{-1} Q_{1}^{-1}\left(1-Q_{0}\right)$ is a pseudo-differential operator of order 1 .

Now it easily follows that the principal symbol of $S_{0}$ is given by

$$
\sigma_{0}\left(S_{0}\right)=i^{-1} \tau_{+}\left(x^{\prime}, \xi^{\prime}\right)
$$

in a local coordinate system such that $x_{n}=0$ on $\Gamma$, if we denote by $\tau_{+}\left(x^{\prime}, \xi^{\prime}\right)$ one root with positive imaginary part of the equation in $t$,

$$
A_{2}\left(x^{\prime}\right) t^{2}+A_{1}^{0}\left(x^{\prime}, \xi^{\prime}\right) t+A_{0}^{0}\left(x^{\prime}, \xi^{\prime}\right)=0
$$

where $A_{1}^{0}$ and $A_{0}^{0}$ are the principal part of $A_{1}$ and $A_{0}$ with respect to $\xi^{\prime}$, respectively, of order 1 and 2 . The inequality (1.5) immediately follows from the fact that $\operatorname{Re} \sigma_{0}\left(S_{0}\right)>0$.

1.2. Let $a_{0}(x)$ and $\gamma_{0}$ be as given in (4) and set

$$
S=a_{0}(x)^{-1}\left(S_{0}-\gamma_{0}\right) .
$$

Then it easily follows from (1.5) that there exist two constants $c_{1}>0$ and $M$ such that the inequality

$$
\operatorname{Re}(S \phi, \phi) \geqq c_{1}\|\phi\|_{1 / 2}^{2}-M\|\phi\|_{0}^{2}
$$

holds for every $\phi \in c^{\infty}(\Gamma)$. Moreover it follows that if $U$ is in $H_{s}(\Omega)$ and satisfies $A U=0$ in $\Omega$, then $u=\left.U\right|_{\Gamma}$ belongs to $H_{s-1 / 2}(\Gamma)$ and $\partial U /\left.\partial \nu\right|_{\Gamma}=S u$, and conversely if $u$ is in $H_{s-1 / 2}(\Gamma)$, then there exists only one $U$ in $H_{s}(\Omega)$ 
such that $A U=0$ in $\Omega,\left.U\right|_{\Gamma}=u$ and $\partial U /\left.\partial \nu\right|_{\Gamma}=S u$. Thus we can prove

Proposition 1.2. Solving in $H_{s}(\Omega)$ the boundary problem (1)-(2) with $F=0$ is equivalent to finding solutions of the equation

$$
(\alpha S+\gamma+\beta) u=f \quad \text { on } \Gamma
$$

in $H_{s-1 / 2}(\Gamma)$.

It can be easily seen from (1.8) that the operator $E=\operatorname{Re} S+M(\operatorname{Re} S$ $\left.=\left(S+S^{*}\right) / 2\right)$ is formally self-adjoint and positive, where $S^{*}$ is the formally adjoint of $S$. Hence there exists the square root $\theta$ of the closure of $E$ in $L^{2}(T)$ and so we have

$$
\operatorname{Re} S=\theta^{2}-M \text {. }
$$

The $\theta$ is also regarded as a pseudo-differential operator of order $1 / 2$ and invertible. The norms $\|\phi\|_{1 / 2}$ and $\|\theta \phi\|_{0}$ are equivalent.

1.3. Let $\rho$ be in $C^{\infty}(\Gamma)$ so that $\rho(x) \geqq 0$ on $\Gamma$. By $\mathscr{U}^{\rho}$ and $\mathscr{F}^{\rho}$ we denote two Hilbert spaces obtained by the completion of $C^{\infty}(\Gamma)$ with respect to the norms

$$
\|u\|=\left(\|\sqrt{\rho} \theta u\|_{0}^{2}+\|u\|_{0}^{2}\right)^{1 / 2}
$$

and

$$
\|f \mid\|^{\prime}=\sup _{u \in \mathscr{u}, u \neq 0} \frac{|(f, u)|}{\|u\|}
$$

respectively. It should be noted that $\mathscr{F}^{\rho}$ is isometric to the dual space of $\mathscr{U}^{\rho}$ and that the multiplication mapping $u \mapsto \phi u$ with $\phi \in C^{\infty}(\Gamma)$ is continuous on $\mathscr{U}^{\rho}$ as well as on $\mathscr{F}^{\rho}$.

Now let $s$ be a real number. By $\mathscr{U}_{s}^{\rho}$ and $\mathscr{F}_{s}^{\rho}$ we denote two Hilbert spaces obtained by the completion of $C^{\infty}(\Gamma)$ with respect to the respective norms

$$
\|u\|_{s}=\left(\sum_{j=1}^{\ell}\left\|T_{s}^{(j)} u\right\|^{2}+\|u\|_{s-1 / 2}^{2}\right)^{1 / 2}
$$

and

$$
\|f\|_{s}^{\prime}=\left(\sum_{j=1}^{\ell}\left\|T_{s}^{(j)} u\right\|^{2}+\|f\|_{s-1}^{2}\right)^{1 / 2},
$$

where $T_{s}^{(j)}(j=1, \cdots, \ell)$ are pseudo-differential operators on $\Gamma$ of order $s$ 
which are defined as follows. We can write the operator $E_{s}$ defined by (1.1) in the form $E_{s}=\tilde{T}_{s}-F_{s}$ where $\tilde{T}_{s}$ is properly supported, $F_{s}$ has a $C^{\infty}$-kernel and $\tilde{T}_{0}$ is the identity, and further assume that for each $j=1$, $\cdots, l$ there exists a compact subset $K_{j}$ of $B_{j}$ such that $\operatorname{supp}\left[\tilde{T}_{s} \phi\right] \subset K_{j}$ for every $\phi \in C_{0}^{\infty}\left(R^{n-1}\right)$ whose support is contained in the compact set supp $\left[\tilde{\zeta}_{j}(y)\right]$ of $B_{j}$. A pseudo-differential operator $T_{s}^{(j)}$ on $\Gamma$ of order $s$ is defined by

$$
\left(T_{s}^{(j)} u\right)(x)=\left\{\begin{array}{cc}
\tilde{T}_{s}\left(\tilde{\zeta}_{j} \tilde{u}\right)\left(\kappa_{j}(x)\right), & x \in \omega_{j} \\
0, & x \notin \omega_{j}
\end{array}\right.
$$

for each $j=1, \cdots, \ell$.

It is easily seen that $\mathscr{U}^{\rho}=\mathscr{U}_{0}^{\rho}$ and $\mathscr{F}^{\rho}=\mathscr{F}_{0}^{\rho}$, since $T_{0}^{(j)} u=\zeta_{j} u$. We can further prove

Proposition 1.3. For all real s,

$$
H_{s-1 / 2}(\Gamma) \supset \mathscr{F}_{s}^{\rho} \supset H_{s}(\Gamma) \supset \mathscr{U}_{s}^{\rho} \supset H_{s+1 / 2}(\Gamma)
$$

is valid with the continuous injections.

Proof. First we note that there exist positive constants $c_{1}, c_{2}$ such that for all $u \in C^{\infty}(\Gamma)$

$$
c_{1}^{-1}\|u\|_{0} \leqq\|u \mid\| \leqq c_{1}\|u\|_{1 / 2}, \quad c_{2}^{-1}\|u\|_{-1 / 2} \leqq\|u\|^{\prime} \leqq c_{2}\|u\|_{0} .
$$

Then it easily follows that $\mathscr{U}_{s}^{\rho} \supset H_{s+1 / 2}(\Gamma)$ and $\mathscr{F}_{s}^{\rho} \supset H_{s}(\Gamma)$ with continuous injections. Now for $u \in C^{\infty}(\Gamma)$, we have

$$
\|u\|_{s}^{2} \leqq \text { const. }\left(\sum_{j=1}^{\ell}\left\|T_{s}^{(j)} u\right\|_{0}^{2}+\|u\|_{s-1 / 2}^{2}\right) \leqq \text { const. }\|u\|_{s}^{2}
$$

and

$$
\|u\|_{s-1 / 2}^{2} \leqq \text { const. }\left(\sum\left\|T_{s}^{(j)} u\right\|_{-1 / 2}^{2}+\|u\|_{s-1}^{2}\right) \leqq \text { const. } \mid\|u\|_{s}^{2} .
$$

These imply $H_{s}(\Gamma) \supset \mathscr{U}_{s}^{\rho}$ and $H_{s-1 / 2} \supset \mathscr{F}_{s}^{\rho}$, respectively.

Q.E.D.

Finally we state two propositions.

Proposition 1.4. Let $L$ be a first order differential operator on $\Gamma$ with $C^{\infty}$-coefficients. Then for every $s$, there exists a constant $C_{s}>0$ such that

$$
\|L(\rho) u\|_{s+1 / 2} \leqq C_{s}\|u\|_{s}
$$

holds for every $u \in C^{\infty}(I)$, where $\rho$ is the function introduced at the beginning of this section. 
Proof. Let $u \in C^{\infty}(\Gamma)$. Then by (1.13) and Lemma A.2, we have

$$
\begin{aligned}
\|L(\rho) u\|_{s+1 / 2} & \leqq C\|\theta L(\rho) u\|_{s} \leqq C\left(\sum_{j=1}^{\ell}\left\|T_{s}^{(j)} \theta L(\rho) u\right\|_{0}+\|\theta L(\rho) u\|_{s-1 / 2}\right) \\
& \leqq C\left(\sum_{j=1}^{\ell}\left\|L(\rho) \theta T_{s}^{(j)} u\right\|_{0}+\|u\|_{s-1 / 2}+\|L(\rho) u\|_{s}\right) \\
& \leqq C\left(\sum_{j=1}^{\ell}\left\|T_{s}^{(j)} u\right\|+\|u\|_{s-1 / 2}+\|L(\rho) u\|_{s}\right) \\
& \leqq C\left(\|u\|_{s}+\|L(\rho) u\|_{s}\right) .
\end{aligned}
$$

where $C$ denotes the various positive constants, from which we can conclude the proposition.

Q.E.D.

Proposition 1.5. Let $L$ be the same as in the preceding proposition. Then for every $s$, there exists a constant $C_{s}>0$ such that

$$
\|L(\rho) u\|_{s+1 / 2}^{\prime} \leqq C_{s}\|u\|_{s}
$$

holds for every $u \in C^{\infty}(\Gamma)$.

Proof. It is enough to prove the inequality

$$
\left\|T_{s+1 / 2}^{(j)}(L(\rho) u)\right\|^{\prime} \leqq \text { const. }\|u\|_{s}
$$

for $j=1, \cdots, \ell$. For any $v \in C^{\infty}(\Gamma)$, we have

$$
\begin{aligned}
\left(T_{s+1 / 2}^{(j)} L(\rho) u, v\right) & =\left(\theta^{-1} T_{s+1 / 2}^{(j)} L(\rho) u, \theta v\right) \\
& =\left(\theta^{-1} T_{s+1 / 2}^{(j)} u, L(\rho) \theta v\right)+\left(\left[\theta^{-1} T_{s+1 / 2}^{(j)}, L(\rho)\right] u, \theta v\right) .
\end{aligned}
$$

Hence by Lemma A.2

$$
\begin{aligned}
\left|\left(T_{s+1 / 2}^{(j)} L(\rho) u, v\right)\right| & \leqq \text { const. }\left(\|u\|_{s}\|\sqrt{\rho} \theta v\|_{0}+\|u\|_{s-1 / 2}\|v\|_{0}\right) \\
& \leqq \text { const. }\|u\|_{s}\|v\| \|
\end{aligned}
$$

which completes the proof.

\section{Chapter 2. The case of type (I)}

2.0. In this chapter, we suppose the manifold $\Gamma_{0}$ to be of type (I). For simplicity, we assume $\alpha \geqq 0$ throughout $\Gamma$. This case was treated also in [4], but the formulation has a little difference.

2.1. The following lemma is nothing but Lemma 4 in [4].

Lemma 2.1. There exists a function $h$ in $C^{\infty}(\Gamma)$ such that $h>0$ on $\Gamma$ and 


$$
\frac{1}{2} \gamma^{*}(h)+h \beta=1 \quad \text { on } \Gamma_{0},
$$

where $\gamma^{*}$ is the adjoint of $\gamma$ defined by the identity

$$
\int_{\Gamma} \gamma u \cdot \bar{v} d \sigma=\int_{\Gamma} u \cdot \overline{\gamma^{*} v} d \sigma, \quad u, v \in C^{\infty}(\Gamma) .
$$

Proof. First note that there exists $C^{\infty}$-function $b(x)$ on $\Gamma$ such that $\gamma^{*}=-\gamma+b(x)$. Then, we have only to find $h$ such that $-\gamma(h)+$ $(b+2 \beta) h=2$ on $\Gamma_{0}$, which is written by the transformation $y=\kappa_{j}(x)$ as $-\partial \tilde{h} / \partial y_{1}+(\tilde{b}+2 \tilde{\beta}) \tilde{h}=2$ in $B_{j}$ when $\omega_{j} \cap \Gamma_{0} \neq \phi$. Let $\tilde{h}=\tilde{h}_{j}$ be a positive solution of this equation. We then define as $h_{j}(x)=\tilde{h}_{j}\left(\kappa_{j}(x)\right)$. On the other hand, on $\omega_{j}$ such that $\omega_{j} \cap \Gamma_{0}=\phi$, we define $h_{j}(x)=1$. The function $h=\sum_{j=1}^{\ell} \zeta_{j} h_{j}$ on $\Gamma$ is the desired one.

Q.E.D.

We are going to consider the equation

$$
h(\alpha S+\gamma+\beta) u=f
$$

instead of treating the equation $(\alpha S+\gamma+\beta) u=f$. Introducing a bilinear form

$$
Q[u, v]=(h(\alpha S+\gamma+\beta) u, v),
$$

we have by (1.9), after simple calculation,

$$
\operatorname{Re} Q[u, u]=(h \alpha \theta u, \theta u)
$$

$$
\begin{aligned}
& +\left(\left(\frac{[[h \alpha, \theta], \theta]}{2}+\frac{\left[h \alpha, S-S^{*}\right]}{4}-h \alpha M\right) u, u\right) \\
& +\left(\left(\frac{1}{2} \gamma^{*}(h)+h \beta\right) u, u\right)
\end{aligned}
$$

for $u \in C^{\infty}(\Gamma)$, where $[A, B]=A B-B A$. Since $h \alpha>0$ on $\Gamma \backslash \Gamma_{0}$, it follows from Lemma 2.1 that there exist a constant $R>0$ such that

$$
R h \alpha+\frac{1}{2} \gamma^{*}(h)+h \beta>0 \quad \text { on } \Gamma \text {. }
$$

Let $H$ be a pseudo-differential operator on $\Gamma$ defined by

$$
H=R h \alpha+h \alpha M-\frac{[[h \alpha, \theta], \theta]}{2}-\frac{\left[h \alpha, S-S^{*}\right]}{4}
$$

and set, for $\varepsilon$ such that $0<\varepsilon \leqq 1$,

$$
Q_{\varepsilon}[u, v]=Q[u, v]+(H u, v)+\varepsilon((S+M) u, v) .
$$

Then it easily follows from (1.9), (2.1) and (2.2) that there exists two positive constants $c_{2}, C$ independent of $\varepsilon$ such that 


$$
\left\{\begin{array}{l}
\operatorname{Re} Q_{\varepsilon}[u, u] \geqq c_{2}\|u\|\left\|^{2}+\varepsilon\right\| \theta u \|_{0}^{2} \\
\left|Q_{\odot}[u, v]\right| \geqq C\|u\|_{1 / 2}\|v\|_{1 / 2},
\end{array}\right.
$$

for all $u, v \in H_{1 / 2}(\Gamma)$. Here and throughout this section, $\|u\| \|$ and $\|f \mid\|^{\prime}$ mean norms (1.10) and (1.11), respectively, with $\rho=h \alpha$. The Lax-Milgram theorem guarantees the existence of $u_{\varepsilon} \in H_{1 / 2}(\Gamma)$ for every $f \in C^{\infty}(\Gamma)$ such that

$$
Q_{\varepsilon}\left[u_{\varepsilon}, v\right]=(f, v), \quad v \in H_{1 / 2}(\Gamma) .
$$

Substitution $v=u_{\varepsilon}$ gives us the inequality

$$
\left\|u_{\varepsilon}\right\| \leqq C_{0}\|f \mid\|^{\prime}
$$

with a constant $C_{0}>0$ not depending on $\varepsilon$. Since $u_{\varepsilon}$ is a weak solution of the elliptic equation, we can assert $u_{\varepsilon} \in C^{\infty}(\Gamma)$, which satisfies the equation

$$
\{h(\alpha S+\gamma+\beta)+H+\varepsilon(S+M)\} u_{\varepsilon}=f \quad \text { on } \Gamma .
$$

TheOREM 2.1. Let $s \geqq \frac{1}{2}$. For every $f \in \mathscr{F}_{s}^{h \alpha}$ we can find one and only one $u \in \mathscr{U}_{s}^{h \alpha}$ satisfying the equation

$$
\{h(\alpha S+\gamma+\beta)+H\} u=f \quad \text { on } \Gamma .
$$

Moreover the inequality

$$
\|u\|_{s} \leqq C_{s}\|f\|_{s}^{\prime}
$$

holds with a constant $C_{s}>0$ independent of $f$.

Proof. First suppose $f \in C^{\infty}(\Gamma)$ and substitute $u=T_{s}^{(j)} u_{\varepsilon}$ (see (1.12) for $T_{s}^{(j)}$ ) in (2.3). Then we have, for $j=1, \cdots, \ell$, by $(2.5)$

$$
\begin{aligned}
c_{2}\left\|T_{s}^{(j)} u_{\varepsilon}\right\|\left\|^{2}+\varepsilon\right\| \theta T_{s}^{(j)} u_{\varepsilon} \|_{0} \leqq & \operatorname{Re} Q_{\varepsilon}\left[T_{s}^{(j)} u_{\varepsilon}, T_{s}^{(j)} u_{\varepsilon}\right] \\
= & \operatorname{Re}\left(\left\{\alpha S+\gamma+\beta+h^{-1} H+\varepsilon h^{-1}(S+M)\right\} T_{s}^{(j)} u_{\varepsilon}, h T_{s}^{(j)} u_{\varepsilon}\right) \\
= & \operatorname{Re}\left(T_{s}^{(j)} h^{-1} f, h T_{s}^{(j)} u_{\varepsilon}\right)+\operatorname{Re}\left(\left[\{\cdots\}, T_{s}^{(j)}\right] u_{\varepsilon}, h T_{s}^{(j)} u_{\varepsilon}\right) \\
= & \operatorname{Re}\left(T_{s}^{(j)} h^{-1} f, h T_{s}^{(j)} u_{\mathrm{\varepsilon}}\right)+\operatorname{Re}\left(\left[\beta+h^{-1} H+\varepsilon h^{-1} M, T_{s}^{(j)}\right] u_{\varepsilon}, h T_{s}^{(j)} u_{\mathrm{\varepsilon}}\right) \\
& \quad+\operatorname{Re}\left(X_{j}+Y_{j}+Z_{j}\right) \\
& \leqq\left\|T_{s}^{(j)} h^{-1} f\right\|\left\|^{\prime}\right\| T_{s}^{(j)} u_{\varepsilon} \|+\operatorname{Re}\left(X_{j}+Y_{j}+Z_{j}\right)+O\left(\|u\|_{s-1 / 2}\right)
\end{aligned}
$$

with

$$
\begin{aligned}
& X_{j}=\left(\left[\alpha S, T_{s}^{(j)}\right] u_{\varepsilon}, h T_{s}^{(j)} u_{\varepsilon}\right), \\
& Y_{j}=\left(\left[\varepsilon h^{-1} S, T_{s}^{(j)}\right] u_{s}, h T_{s}^{(j)} u_{\varepsilon}\right), \\
& Z_{j}=\left(\left[\gamma, T_{s}^{(j)}\right] u_{\varepsilon}, h T_{s}^{(j)} u_{\varepsilon}\right) .
\end{aligned}
$$


In this section, we denote generally by $C$ various constants independent of $\varepsilon, 0<\varepsilon \leqq 1$, and write, for brevity, as $u=u_{\varepsilon}$ and $T=T_{s}^{(j)}$ in the below. Since

$$
X_{j}=\left(\theta^{-1}[S, T] u, \theta h \alpha T u\right)+([\alpha, T] S u, h T u)
$$

it easily follows from Lemmas A.1 and A.2 that

$$
\left|X_{j}\right| \leqq C\|u\|_{s-1 / 2}\left(\|h \alpha \theta T u\|_{0}+\|\sqrt{\alpha} \theta T u\|_{0}+\|u\|_{s-1 / 2}\right) .
$$

Accordingly, for every $\delta>0$ there exists a constant $C_{\delta}>0$ such that

$$
\left|X_{j}\right| \leqq \delta\|\sqrt{h \alpha} \theta T u\|_{0}^{2}+C_{\delta}\|u\|_{s-1 / 2}^{2} .
$$

Similarly, since

$$
Y_{j}=\left(\theta^{-1}\left[\varepsilon h^{-1} S, T\right] u, \theta h T u\right),
$$

we obtain

$$
\left|Y_{j}\right| \leqq \varepsilon C\|u\|_{s-1 / 2}\|\theta T u\|_{0} \leqq \varepsilon\left(\delta\|\theta T u\|_{0}^{2}+C_{\delta}\|u\|_{s-1 / 2}^{2}\right) .
$$

Thus we have

$$
\begin{gathered}
c_{2}\|T u\|^{2}+\varepsilon\|\theta T u\|_{0}^{2} \leqq\left.\|T f\|\right|^{\prime}\|T u\|+\delta\|\sqrt{h \alpha} \theta T u\|_{0}^{2} \\
+\delta \varepsilon\|\theta T u\|_{0}^{2}+C_{\delta}\|u\|_{s-1 / 2}^{2}+\operatorname{Re} Z_{j}
\end{gathered}
$$

which implies

$$
\|T u\|^{2} \leqq C\left(\|T f\|^{2}+\|u\|_{s-1 / 2}^{2}+\operatorname{Re} Z_{j}\right)
$$

Consequently,

$$
\|u\|_{s}^{2} \leqq C\left(\|f \mid\|_{s}^{2}+\|u\|_{s-1 / 2}^{2}+\sum_{j=1}^{\ell} \operatorname{Re} Z_{j}\right) .
$$

Now we shall show the existence of a constant $C_{\delta}$ for any $\delta>0$ such that

$$
\sum_{j=1}^{\ell} \operatorname{Re} Z_{j} \leqq \delta\|\| u\left\|_{s}^{2}+C_{\delta}\right\| u \|_{s-1 / 2}^{2} .
$$

On $\omega_{j}$ such that $\omega_{j} \cap \Gamma_{0} \neq \phi$, the operator $[\gamma, T]$ is transformed by $\kappa_{j}$ to

$$
\begin{aligned}
{\left[\frac{\partial}{\partial y_{1}}, \tilde{T}_{s} \tilde{\zeta}_{j}\right] } & =\left[\frac{\partial}{\partial y_{1}}, \tilde{T}_{s}\right] \tilde{\zeta}_{j}+\tilde{T}_{s}\left[\frac{\partial}{\partial y_{1}}, \tilde{\zeta}_{j}\right] \\
& =\left[\frac{\partial}{\partial y_{1}}, E_{s}+F_{s}\right] \tilde{\zeta}_{j}+\tilde{T}_{s} \frac{\partial \tilde{\zeta}_{j}}{\partial y_{1}}=\left[\frac{\partial}{\partial y_{1}}, F_{s}\right] \tilde{\zeta}_{j}+\tilde{T}_{s} \frac{\partial \tilde{\zeta}_{j}}{\partial y_{1}}
\end{aligned}
$$


since $T=T_{s}^{(j)}$ and $\left[\partial / \partial y_{1}, E_{s}\right]=0$. Hence we have

$$
\left|Z_{j}\right| \leqq C\left(\left\|\gamma\left(\zeta_{j}\right) u\right\|_{s}+\|u\|_{s-1 / 2}\right)\|u\|_{s} .
$$

From the fact that $\gamma\left(\zeta_{j}\right)=0$ in some neighborhood of $\Gamma_{0}$, it follows by (1.13) that

$$
\begin{aligned}
\left\|\gamma\left(\zeta_{j}\right) u\right\|_{s} \leqq C\|h \alpha u\|_{s} & \leqq C\left(\sum_{i=1}^{\ell}\|T h \alpha u\|_{0}+\|u\|_{s-1 / 2}\right) \\
& \leqq C\left(\sum_{\imath=1}^{\ell}\|h \alpha T u\|_{0}+\|u\|_{s-1 / 2}\right) .
\end{aligned}
$$

Using the interpolation inequality, we can assert the existence of $C_{\delta}$, for any $\delta>0$, such that

$$
\left\|\gamma\left(\zeta_{j}\right) u\right\|_{s} \leqq \delta \sum_{i=1}^{\ell}\|\theta h \alpha T u\|_{0}+C_{\delta}\|u\|_{s-1 / 2} .
$$

On the other hand, on $\omega_{j}$ such that $\omega_{j} \cap \Gamma_{0}=\phi$ we can immediately obtain

$$
\left|Z_{j}\right| \leqq C\left(\left\|\gamma\left(\zeta_{j}\right) u\right\|_{s}+\left\|\zeta_{j} u\right\|_{s}\right)\|u\|_{s} .
$$

Since $\zeta_{j}$ as well as $\gamma\left(\zeta_{j}\right)$ vanishes near $\Gamma_{0}$, we can estimate $\left\|\gamma\left(\zeta_{j}\right) u\right\|_{s}$ and $\mid \zeta_{j} u \|_{s}$ as in (2.8). Thus we can establish (2.7), and hence (2.6) becomes

$$
\|u\|_{s} \leqq C\left(\|f\|_{s}^{\prime}+\|u\|_{s-1 / 2}\right) .
$$

If $s \geqq 1 / 2$, it then follows from (2.4) and the interpolation inequality that for any $\delta>0$ there exists a constant $C_{\delta}>0$ such that

$$
\|u\|_{s-1 / 2} \leqq \delta\|u\|_{s}+C_{\delta}\|f\|_{s}^{\prime},
$$

which together with (2.9) implies the inequality

$$
\left\|u_{\varepsilon}\right\|_{s} \leqq C_{s}\|f\|_{s}^{\prime}
$$

or all $s \geqq 1 / 2$ with a constant $C_{s}>0$ independent of $\varepsilon$ and $f$, where we jut again $u=u_{\varepsilon}$.

By (2.10), we can choose a sequence $\varepsilon_{1}>\varepsilon_{2}>\cdots \rightarrow 0$ such that $u_{\varepsilon_{j}}$ zonverges in $C^{\infty}(\Gamma)$. Let $u$ be the limit function. Then we have from 2.5) and (2.10)

$$
\left\{\begin{array}{l}
h(\alpha S+\gamma+\beta)+H) u=f \quad \text { on } \Gamma \\
\|u\|_{s} \leqq C_{s}\|f\|_{s}^{\prime} .
\end{array}\right.
$$

Now let $f \in \mathscr{F}_{s}^{h \alpha}(s \geqq 1 / 2)$ and choose $f_{j}$ in $C^{\infty}(\Gamma)$ so that $f_{j} \rightarrow f$ in $\mathscr{F}_{s}^{h \alpha}$ as 
$j \rightarrow \infty$. We have just proved that, for each $f_{j}$, there exists $u_{j} \in C^{\infty}(\Gamma)$ satisfying (2.11) with $u=u_{j}$ and $f=f_{j}$. It is not hard to prove that the sequence $u_{f}$ has a limit $u$ belonging to $\mathscr{U}_{s}^{h \alpha}$ and satisfying (2.11).

To complete the proof, we must show the uniqueness. Let $u$ be a solution in $\mathscr{U}_{s}^{h \alpha}(s \geqq 1 / 2)$ of the equation $\{h(\alpha S+\gamma+\beta)+H\} u=0$ on $\Gamma$. It follows from Proposition 1.3 that $u \in H_{1 / 2}(\Gamma)$. Hence, by (2.3) with $\varepsilon=0$, we have

$$
0=(h(\alpha S+\gamma+\beta) u+H u, u)=Q_{0}[u, u]=c_{2}\|u\|^{2} .
$$

This implies $u=0$.

2.2. If we write the solution $u$ in Theorem 2.1 as $u=K f$, then $K$ is a continuous mapping of $\mathscr{F}_{s}^{h \alpha}$ into $\mathscr{U}_{s}^{h \alpha}(s \geqq 1 / 2)$ and satisfies

$$
(h(\alpha S+\gamma+\beta)+H) K=1 \quad \text { on } \mathscr{F}_{s}^{h \alpha} .
$$

Proposition 1.3 guarantees that $K$ is also a continuous mapping of $H_{s}(\Gamma)$ $(s \geqq 1 / 2)$ into itself. Let $f$ be in $H_{s}(\Gamma)$. If $g \in H_{s}(\Gamma)$ and satisfies

$$
(1-H K) g=f,
$$

then $u=K g$ satisfies

$$
h(\alpha S+\gamma+\beta) u=f .
$$

Conversely, if $u \in H_{s}(\Gamma)$ and satisfies (2.13), we have $h(\alpha S+\gamma+\beta) u+H u$ $=f+H u$. Therefore $u=K(f+H u)$. Put $g=f+H u$. Then $h(\alpha S+\gamma$ $+\beta) K g=f$. So we have (2.12). Thus it is enough to treat the equation (2.12) in order to solve the equation (2.13). If $g \in H_{s}(\Gamma)$, then $K g \in \mathscr{U}_{s}^{h \alpha}$. Using Proposition 1.4, Lemmas A.1 and A.2, we have $H K g \in H_{s+1 / 2}(\Gamma)$. Moreover it easily follows that $H K$ is a continuous mapping of $H_{s}(\Gamma)$ into $H_{s+1 / 2}(\Gamma)$. Accordingly, $H K$ is a compact operator on $H_{s}(\Gamma)$. Applying the Riesz-Schauder theory, we can establish the main theorem of this section.

TheOREM 2.2. (i) Let $s \geqq 1 / 2$ and $f \in H_{s}(\Gamma)$. Then the equation (2.13) admits a solution $u \in H_{s}(\Gamma)$ if and only if $f$ is orthogonal to a finite-dimensional subspaces $\tilde{N}_{s}$ of $H_{s}(\Gamma)$, which has the same dimension as $N=\{u \in$ $\left.H_{s}(\Gamma) ;(\alpha S+\gamma+\beta) u=0\right\}$. (ii) Every solution $u \in H_{s}(\Gamma)(s \geqq 1 / 2)$ of (2.13) belongs to $H_{t}(\Gamma)$ if $f \in H_{t}(\Gamma)$ with $t>s$.

Proof. For the proof of (i), see pp. 284-5 of [9]. So we shall prove 
only (ii). Let $f$ be in $H_{t}(\Gamma)$ with $t>s$. If $u \in H_{s}(\Gamma)$ and satisfies (2.13), then $h(\alpha S+\gamma+\beta) u+H u=f+H u$. By Proposition $1.5(\rho=h \alpha)$, we have $H u \in \mathscr{F}_{s=1 / 2}^{h \alpha}$. Therefore $u=K f+K H u \in H_{t}(\Gamma)$ if $t \leqq s+1 / 2$, and $u \in$ $H_{s+1 / 2}(\Gamma)$ if $t>s+1 / 2$. If the later takes place, we have only to repeat the above process.

Q.E.D.

Remark 2.1. The dimension of $N$ is independent of $s$. In fact, by virtue of Theorem 2.2 (ii), we have $N \subset C^{\infty}(\Gamma)$.

2.3. We shall study the possibility of $\operatorname{dim} N=0$ in the preceding theorem. For this purpose we first state a lemma which is similar to Lemma 2 in [4]. Using Lemma 2.1, we can easily prove it.

Lemma 2.2. We can find a function $q(x) \in C^{\infty}(\bar{\Omega})$ satisfying

(i) $q(x)>0$ in $\Omega$ and $q(x)=h(x) \alpha(x)$ on $\Gamma$,

(ii) there exist two positive constants $k$ and $d$ such that

$$
k \operatorname{dis}(x, \Gamma) \leqq q(x) \quad \text { in } \Omega_{d}=\{x \in \bar{\Omega} ; \operatorname{dis}(x, \Gamma)<d\},
$$

(iii) the inequality

$$
\frac{1}{2} \frac{\partial q}{\partial \nu}+\frac{1}{2} \gamma^{*}(h)+h \beta \geqq c_{3}
$$

holds on $\Gamma$ with a constant $c_{3}>0$.

Now we consider a bilinear form

$$
\begin{aligned}
B[U, V]= & \int_{\Omega}\left(\sum_{i, j=1}^{n} a_{i j} \partial_{j} U \cdot \overline{\partial_{i}}(q V)+\sum_{i=1}^{n} b_{i} \partial_{i} U \cdot \overline{q V}+c U \cdot \overline{q V}\right) d x \\
& +\int_{\Gamma} h(\gamma u+\beta u) \cdot \bar{v} d \sigma,
\end{aligned}
$$

with $u=\left.U\right|_{\Gamma}$ and $v=\left.V\right|_{\Gamma}$. Integrating by part, we obtain

$$
B[U, V]=\int_{\Omega} q A U \cdot \bar{V} d x+\int_{\Gamma} h\left(\alpha \frac{\partial U}{\partial \nu}+\gamma u+\beta u\right) \cdot \bar{v} d \sigma .
$$

On the other hand, by (3) we have

$$
\begin{aligned}
\operatorname{Re} B[U, U]= & \operatorname{Re} \int_{\Omega} q\left(\Sigma a_{i j} \partial_{j} U \cdot \overline{\partial_{i} U}+\Sigma b_{i} \partial_{i} U \cdot \bar{U}+c U \bar{U}\right) d x \\
& +\frac{1}{2} \int_{\Omega} \sum a_{i j} \partial_{i} q \partial_{j}(U \bar{U}) d x+\int_{\Gamma}\left(\frac{1}{2} \gamma^{*}(h)+h \beta\right) u \cdot \bar{u} d \sigma
\end{aligned}
$$




$$
\begin{aligned}
& \geqq \frac{c_{0}}{2}\|p \partial U\|_{0, \Omega}^{2}+\int_{\Omega}(c-\mu)|p U|^{2} d x+\frac{1}{2} \int_{\Omega} A_{0} q \cdot|U|^{2} d x \\
& +\int_{\Gamma}\left(\frac{1}{2} \frac{\partial q}{\partial \nu}+\frac{1}{2} \gamma^{*}(h)+h \beta\right)|u|^{2} d \sigma
\end{aligned}
$$

where $\mu$ is a constant, $p(x)=\sqrt{q(x)}, A_{0} q=-\sum_{i, j=i}^{n} \partial_{i}\left(a_{i j} \partial_{j} q\right)$ and

$$
\|p \partial U\|_{0, \Omega}^{2}=\sum_{j=1}^{n} \int q\left|\partial_{j} U\right|^{2} d x
$$

It then follows from Lemma 2.2 and Lemma A.3 that there exist two constants $c_{4}>0$ and $\lambda_{0}$ such that

$$
\operatorname{Re} B[U, U] \geqq c_{4}\|p \partial U\|_{0, \Omega}^{2}-\lambda_{0}\|p U\|_{0, \Omega}^{2}+c_{3}\|u\|_{0}^{2}
$$

for all $U \in C^{\infty}(\bar{\Omega})$.

Denote by $S_{2}$ the operator $S$ corresponding to the operator $A_{2}=A$ $+\lambda$. Then we have

Theorem 2.3. Let $N(\lambda)=\left\{u \in H_{s}(\Gamma)(s \geqq 1 / 2) ;\left(\alpha S_{2}+\gamma+\beta\right) u=0\right\}$. Then $\operatorname{dim} N(\lambda)=0$ for all $\lambda \geqq \lambda_{0}$.

Proof. In view of Theorem 2.2 (ii), we can immediately prove that $N_{\lambda} \subset C^{\infty}(\Gamma)$. Let $U$ be a $C^{\infty}$-solution of the Dirichlet problem; $A_{\lambda} U=0$ in $\Omega$ and $U=u$ on $\Gamma$ with $u \in N(\lambda)$. From Proposition 1.2, it follows $\alpha \partial U / \partial \nu+\gamma U+\beta U=0$ on $\Gamma$. This implies $U=0$, if we apply (2.14) and (2.15) with this $U$. So we have $u=0$.

2.4. Finally we return to the original problem (1)-(2). Corresponding to Theorem 2.2, we can state

THEOREM 2.4. (i) Let $s \geqq 0$ and $(F, f)$ belong to $H_{s}(\Omega) \times H_{s+1 / 2}(\Gamma)$. Then the problem

$$
\left\{\begin{array}{l}
A U=F \quad \text { in } \Omega \\
\alpha \frac{\partial U}{\partial \nu}+\gamma U+\beta U=f \quad \text { on } \Gamma
\end{array}\right.
$$

admits a solution $U \in H_{s+1}(\Omega)$ if and only if $(F, f)$ is orthogonal to a finitedimensional subspace of $H_{s}(\Omega) \times H_{s+1 / 2}(\Gamma)$, and the space of solutions of (2.16) with $F=f=0$ has the finite dimension. (ii) If $(F, f) \in H_{t}(\Omega) \times$ $H_{t+1 / 2}(\Gamma)(t>s \geqq 0)$, every solution $U \in H_{s+1}(\Omega)$ of $(2.15)$ belongs to $H_{t+1}(\Omega)$. 
Proof. (i) Let $V=V_{F}$ be the unique solution in $H_{s+2}(\Omega)$ of the Dirichlet problem; $A V=F$ in $\Omega$ and $V=0$ on $\Gamma$. Then $v^{\prime}=\partial V /\left.\partial \nu\right|_{\Gamma}$ is in $H_{s+1 / 2}(\Gamma)$ and the inequality

$$
\left\|v^{\prime}\right\|_{s+1 / 2} \leqq C\|V\|_{s+2, \Omega} \leqq C\|F\|_{s, \Omega}
$$

holds for every $F \in H_{s}(\Omega)$. Applying Theorem 2.2 (i), we can find a solution $u \in H_{s+1 / 2}(\Gamma)$ of the equation

$$
(\alpha S+\gamma+\beta) u=f-\alpha v^{\prime}
$$

if and only if $h\left(f-\alpha v^{\prime}\right)$ is orthogonal to $\tilde{N}_{s+1 / 2}$.

Let $u$ be a solution in $H_{s+1 / 2}(T)$ of (2.18). It then follows from Propositions 1.1 and 1.2 that a solution $W$ of the Dirichlet problem, $A W=0$ in $\Omega$ and $W=u$ on $\Gamma$, satisfies

$$
\alpha \frac{\partial W}{\partial \nu}+\gamma W+\beta W=f-\alpha v^{\prime}
$$

on $\Gamma$. We can easily see that $U=V+W$ satisfies (2.16). Conversely, let $U \in H_{s+1}(\Omega)$ be a solution of the problem (2.16) and $V=V_{F}$ be the same function as above. Then it follows that $W=U-V$ satisfies $A W$ $=0$ in $\Omega$ and (2.19). Hence $u=\left.W\right|_{\Gamma}$ is in $H_{s+1 / 2}(\Gamma)$ and satisfies (2.18). Thus we showed the problem (2.16) admits a solution in $H_{s+1}(\Omega)$ if and only if $h\left(f-\alpha v^{\prime}\right)$ is orthogonal to $\tilde{N}_{s+1 / 2}$. By (2.17), the linear mapping $(F, f) \mapsto f-\alpha v^{\prime}$ is a continuous operator of $H_{s}(\Omega) \times H_{s+1 / 2}(\Gamma)$ into $H_{s+1 / 2}(\Gamma)$. Hence there exists a finite number of linear functionals $\Phi_{i}$ on $H_{s}(\Omega) \times$ $H_{s+1 / 2}(\Gamma)$ such that (2.16) admits a solution in $H_{s+1}(\Omega)$ if and only if $\Phi_{i}(F, f)=0$ for all $i$. Now if $U$ satisfies (2.16) with $F=0$ and $f=0$, then we have $(\alpha S+\gamma+\beta) u=0\left(u=\left.U\right|_{\Gamma}\right)$. These complete the proof of (i).

(ii) Let $(F, f) \in H_{t}(\Omega) \times H_{t+1 / 2}(\Gamma)$ and $U$ be a solution in $H_{s+1}(\Omega)$ of (2.16) Set $W=U-V$. Here $V=V_{F}$ and note that $V \in H_{t+2}(\Omega)$. Then $w=\left.W\right|_{\Gamma}$ satisfies (2.18), where $f-\alpha v^{\prime}$ belongs to $H_{t+1 / 2}(\Gamma)$. According to Theorem 2.2 (ii), we have $w \in H_{t+1 / 2}(\Gamma)$, which proves $W \in H_{t+1}(\Omega)$. Hence $U \in H_{t+1}(\Omega)$.

Q.E.D.

As a corollary of Theorems 2.3 and 2.4, we can prove

THEOREM 2.5. Let $\lambda_{0}$ be the number introduced in (2.15) and $\lambda$ be any real number such that $\lambda \geqq \lambda_{0}$. Then for every $(F, f) \in H_{s}(\Omega) \times H_{s+1 / 2}(\Gamma)$ $(s \geqq 0)$, we can find one and only one $U \in H_{s+1}(\Omega)$ satisfying 


$$
\left\{\begin{array}{l}
(A+\lambda) U=F \quad \text { in } \Omega \\
\alpha \frac{\partial U}{\partial \nu}+\gamma U+\beta U=f \quad \text { on } \Gamma .
\end{array}\right.
$$

Moreover the inequality

$$
\|U\|_{s+1, \Omega} \leqq C_{s}\left(\|F\|_{s, \Omega}+\|f\|_{s+1 / 2}\right)
$$

holds with a suitable constant $C_{s}>0$.

Proof. Let $\lambda \geqq \lambda_{0}$. Theorem 2.2 with $S=S_{\lambda}$ and Theorem 2.3 guarantee that for every $(F, f) \in H_{s}(\Omega) \times H_{s+1 / 2}(\Gamma)(s \geqq 0)$, the equation (2.20) has one and only one solution $U$ in $H_{s+1}(\Omega)$. Now we set $W=U-V$. Then it follows that $w=\left.W\right|_{\Gamma}$ satisfies (2.18) and is estimated by

$$
\|w\|_{s+1 / 2} \leqq \text { const. }\left\|f-\alpha v^{\prime}\right\|_{s+1 / 2} \leqq \text { const. }\left(\|F\|_{s, \Omega}+\|f\|_{s+1 / 2}\right) \text {. }
$$

Therefore by (2.17), we have

$$
\|U\|_{s+1, \Omega} \leqq\|V\|_{s+1, \Omega}+\|W\|_{s+1, \Omega} \leqq \text { const. }\left(\|F\|_{s, \Omega}+\|W\|_{s+1 / 2}\right),
$$

which completes the proof.

\section{Chapter 3. The case of type (II)}

3.0. This chapter is devoted to the manifold $\Gamma_{0}$ of type (II). Suppose that $\Gamma_{0}$ is a closed manifold which devides $\Gamma$ into two open sets $\Gamma_{-}, \Gamma_{+}$ so that $\alpha<0$ on $\Gamma_{-}, \alpha>0$ on $\Gamma_{+}$and $\alpha=0$ on $\Gamma_{0}$. We then consider the boundary condition $\left(2^{\prime \prime}\right)$ with $\gamma$ transversal from $\Gamma_{-}$to $\Gamma_{+}$on $\Gamma_{0}$.

3.1. After Lemma 2.1, we first introduce an auxiliary function $h$. Note that this $h$ is different from $h$ in Lemma 2.1 .

Lemma 3.1. There exists a function $h$ in $C^{\infty}(\Gamma)$ such that

(i) $h<0$ on $\Gamma_{-}, h>0$ on $\Gamma_{+}$and $h=0$ on $\Gamma_{0}$,

(ii) $\gamma(h)=1$ near $\Gamma_{0}$,

(iii) on $\omega_{j}$ such that $\omega_{j} \cap \Gamma_{0} \neq \phi, h(x)$ is transformed to $y_{1}$ by $\kappa_{j}$, i.e. $h\left(\kappa_{j}^{-1}(y)\right)=y_{1}$ on the ball $B_{j}$ in $R^{n-1}$ with the origin as center.

Proof. Setting $y=\kappa_{j}(x)$, we define

$$
h(x)=\sum_{j=1}^{\ell} \zeta_{j}(x) h_{j}(x),
$$

where $h_{j}(x)=y_{1}$ on $\omega_{j}$ such that $\omega_{j} \cap \Gamma_{0} \neq \phi,=1$ on $\omega_{j}$ such that $\omega_{j} \subset$ 
$\Gamma_{+}$and $=-1$ on $\omega_{j}$ such that $\omega_{j} \subset \Gamma_{-}$. Then (i) and (iii) are obvious. Since $\gamma\left(\zeta_{j}\right)=0$ near $\Gamma_{0}$ and $\gamma\left(h_{j}\right)=1$ on $\omega_{j}$ for all $j$ such that $\omega_{j} \cap \Gamma_{0} \neq$ $\phi$, we have $\gamma(h)=\sum_{j=1}^{\ell} \zeta_{j} \gamma\left(h_{j}\right)=1$ near $\Gamma_{0}$, which prove (ii).

Q.E.D.

Now we consider the equation

$$
(\alpha S+\gamma+\beta) h u=f
$$

instead of $(\alpha S+\gamma+\beta) u=f$. We can then obtain similar results as in Theorems 2.1, 2.2 and 2.3. To do so, we introduce a bilinear form

$$
Q[u, v]=\left(\left(h \alpha S+h \gamma+\beta_{0}\right) u, v\right),
$$

where $\beta_{0}$ is defined by

$$
(\alpha S+\gamma+\beta) h=h \alpha S+h \gamma+\beta_{0},
$$

i.e., $\beta_{0}=\gamma(h)+h \beta+\alpha[S, h]$. By the same way as in (2.1), we have

$$
\operatorname{Re} Q[u, u]=(h \alpha \theta u, \theta u)
$$

$$
\begin{aligned}
& +\left(\left(\frac{[[h \alpha, \theta], \theta]}{2}+\frac{\left[h \alpha, S-S^{*}\right]}{4}-h \alpha M\right) u, u\right) \\
& +\left(\left(\frac{1}{2} \gamma^{*}(h)+\frac{\beta_{0}+\beta_{0}^{*}}{2}\right) u, u\right) .
\end{aligned}
$$

LEMmA 3.2. There exist two positive constants $R$ and $c$ such that

$$
\left(\left(R h \alpha+\frac{1}{2} \gamma(h)+\frac{1}{2} h b+h \beta\right) u, u\right)-|(\alpha[S, h] u, u)| \geqq c\|u\|_{0}^{2}, \quad u \in C^{\infty}(\Gamma),
$$

where $b$ is $a C^{\infty}$-function on $\Gamma$ defined by $\gamma^{*}=-\gamma+b$.

Proof. Since $h \alpha>0$ in $\Gamma \backslash \Gamma_{0}, \gamma(h)=1$ on $\Gamma_{0}$ and $h=0$ on $\Gamma_{0}$, we can find two positive constants $R_{0}$ and $c$ such that

$$
R_{0} h \alpha+\frac{1}{2} \gamma(h)+\frac{1}{2} h b+h \beta \geqq 2 c \quad \text { on } \Gamma .
$$

Accordingly, we have

$$
\begin{aligned}
& \left|\left(\left(\left(R_{0}+R_{1}\right) h \alpha+\frac{1}{2} \gamma(h)+\frac{1}{2} h b+h \beta\right) u, u\right)\right| \geqq 2 c\|u\|_{0}^{2}+R_{1} \int_{\Gamma} h \alpha|u|^{2} d \sigma \\
& \geqq 2 c\|u\|_{0}^{2}+R_{1} \min _{\Gamma-V}(h \alpha)\|u\|_{0, \Gamma-V}^{2},
\end{aligned}
$$

where $V$ is a neighborhood of $\Gamma_{0}$ and $R_{1}$ is any positive constant. Taking $V$ as $V_{\delta}=\{x \in \Gamma ;|\alpha(x)|<\delta\}$ for $\delta>0$, we can establish, for all $u \in C^{\infty}(\Gamma)$,

$$
\left|\int_{\Gamma} \alpha[S, h] u \cdot \bar{u} d \sigma\right| \leqq K_{0}\|u\|_{0}\left(\|\alpha u\|_{0, V_{\delta}}+\|\alpha u\|_{0, \Gamma-V_{\delta}}\right)
$$




$$
\begin{aligned}
& \leqq K_{\text {n }}\|u\|_{0}\left(\delta\|u\|_{0}+\max _{\Gamma}|\alpha| \cdot\|u\|_{0, \Gamma-V_{\delta}}\right) \\
& \leqq 2 K_{0} \delta\|u\|_{0}^{2}+\frac{K_{0}}{4 \delta} \max _{\Gamma}|\alpha| \cdot\|u\|_{0, \Gamma-V_{\delta}}^{2},
\end{aligned}
$$

where $K_{0}$ is positive constant such that

$$
\|[S, h] u\|_{0} \leqq K_{0}\|u\|_{0}, \quad u \in C^{\infty}(\Gamma) .
$$

Choosing $\delta=c / 2 K_{0}$ and

$$
R_{1}=\frac{K_{0} \max _{\Gamma}|\alpha|}{4 \delta \min _{\Gamma-v_{\delta}}(\alpha h)}
$$

we can conclude the lemma, with $R=R_{0}+R_{1}$.

Q.E.D.

Let $H$ be a pseudo-differential operator on $\Gamma$ defined by

$$
H=R h \alpha+h \alpha M-\frac{[[h \alpha, \theta], \theta]}{2}-\frac{\left[h \alpha, S-S^{*}\right]}{4}
$$

and set, for $\varepsilon$ such that $0<\varepsilon \leqq 1$,

$$
\mathrm{Q}_{\varepsilon}[u, v]=Q[u, v]+(H u, v)+\varepsilon((S+M) u, v) .
$$

It then follows from (3.1) that

$$
\begin{aligned}
\operatorname{Re} Q_{\varepsilon}[u, u]= & (h \alpha \theta u, \theta u)+\left(\left(\frac{1}{2} \gamma^{*}(h)+\frac{\beta_{0}+\beta_{0}^{*}}{2}+R h \alpha\right) u, u\right)+\varepsilon\|\theta u\|_{0} \\
& \geqq(h \alpha \theta u, \theta u)+\left(\left(R h \alpha+\frac{1}{2} \gamma(h)+\frac{1}{2} h b+h \beta\right) u, u\right) \\
& -|(\alpha[S, h] u, u)|+\varepsilon\|\theta u\|_{0}^{2} .
\end{aligned}
$$

Therefore, by Lemma 3.2 we have

$$
\operatorname{Re} Q_{\varepsilon}[u, u] \geqq(h \alpha \theta u, \theta u)+c\|u\|_{0}^{2}+\varepsilon\|\theta u\|_{0}^{2} \geqq c_{2}\|\omega\|^{2}+\varepsilon\|\theta u\|_{0}^{2}
$$

with a suitable constant $c_{2}>0$. Using the same argument as in $\S 2$, we can obtain $u_{\varepsilon} \in C^{\infty}(\Gamma)$ for every $f \in C^{\infty}(\Gamma)$ such that $\left\|u_{\varepsilon}\right\| \leqq C_{0}\|f\|^{\prime}$ and

$$
\{(\alpha S+\gamma+\beta) h+H+\varepsilon(S+M)\} u_{\varepsilon}=f \quad \text { on } \Gamma \text {. }
$$

Theorem 3.1. Let $s \geqq 1 / 2$. For every $f \in \mathscr{F}_{s}^{h \alpha}$ we can find one and only one $u \in \mathscr{U}_{s}^{h \alpha}$ satisfying the equation

$$
\{(\alpha S+\gamma+\beta) h+H\} u=f \quad \text { on } \Gamma .
$$

Moreover the inequality 


$$
\|u\|_{s} \leqq C_{s}\|f\|_{s}^{\prime}
$$

holds with a constant $C_{s}>0$ independent of $f$.

Proof. Suppose $f \in C^{\infty}(\Gamma)$ and substitute $u=T_{s}^{(j)} u_{\varepsilon}(s \geqq 1 / 2)$ in (3.3), which we write, for simplicity, as $T u$ in the below. If we go through the same procedure as in the proof of Theorem 2.1, it follows from (3.4) that for each $j=1, \cdots, \ell$

$$
\begin{aligned}
& c_{2}\|T u\|^{2}+\varepsilon\|\theta T u\|_{0}^{2} \leqq \operatorname{Re}(T f, T u)+\operatorname{Re}\left(\left[\beta_{0}+H+\varepsilon M, T\right] u, T u\right) \\
& \quad+\operatorname{Re}([h \alpha S+h \gamma+\varepsilon S, T] u, T u) \\
& \quad \leqq\|T f\|\left\|^{\prime}\right\| T u\left\|+\operatorname{Re}\left(X_{j}+Y_{j}+Z_{j}\right)+C\right\| u \|_{s-1 / 2}
\end{aligned}
$$

with

$$
\left\{\begin{array}{l}
X_{j}=([h \alpha S, T] u, T u) \\
Y_{j}=([\varepsilon S, T] u, T u) \\
Z_{j}=([h \gamma, T] u, T u)
\end{array},\right.
$$

and that for every $\delta>0$ there exists a constant $C_{\delta}>0$ such that

$$
\left\{\begin{array}{l}
\left|X_{j}\right| \leqq \delta\|\sqrt{h \alpha} \theta T u\|_{0}^{2}+C_{\delta}\|u\|_{s-1 / 2}^{2} \\
\left|Y_{j}\right| \leqq \varepsilon\left(\delta\|\theta T u\|_{0}^{2}+C_{\delta}\|u\|_{s-1 / 2}^{2}\right) \\
|(h[\gamma, T] u, T u)| \leqq \delta\|u\|_{s}^{2}+C_{\delta}\|u\|_{s-1 / 2}^{2}
\end{array}\right.
$$

from which we can easily deduce

$$
\|u\|_{s}^{2} \leqq C\left(\|f\|_{s}^{2}+\|u\|_{s-1 / 2}^{2}+\operatorname{Re} \sum_{j=1}^{l}([h, T] \gamma u, T u)\right)
$$

Here and in the following, the letters $C, C_{0}, C_{1} \cdots$ stand for positive constants.

Now we shall estimate the last term of (3.6). On $\omega_{j}$ such that $\omega_{j} \cap$ $\Gamma_{0} \neq \phi$, the operator $\left[h, T_{s}^{(j)}\right] \gamma$ is transformed by $\kappa_{j}$ to

$$
\begin{aligned}
{\left[y_{1}, \tilde{T}_{s} \tilde{\zeta}_{j}\right] \frac{\partial}{\partial y_{1}} } & =\left[y_{1}, E_{s} \tilde{\zeta}_{j}\right] \frac{\partial}{\partial y_{1}}+\left[y_{1}, F_{s} \tilde{\zeta}_{j}\right] \frac{\partial}{\partial y_{1}} \\
& =\left[y_{1}, E_{s}\right] \frac{\partial}{\partial y_{1}} \cdot \tilde{\zeta}_{j}-\left[y_{1}, E_{s}\right] \frac{\partial \tilde{\zeta}_{j}}{\partial y_{1}}+\left[y_{1}, F_{s} \tilde{\zeta}_{j}\right] \frac{\partial}{\partial y_{1}} \\
& =s\left(\frac{\partial}{\partial y_{1}}\right)^{2} E_{s-2} \tilde{\zeta}_{j}+p . d .0 . \text { of order } s-1 .
\end{aligned}
$$

Since $\left.E_{s}=\left(1-\Delta_{y}\right) E_{s-2}\left(\Delta_{y}\right)=\sum_{i=1}^{n}\left(\partial / \partial y_{i}\right)^{2}\right)$, we have 


$$
\begin{aligned}
([h, T] \gamma u, T u) & =s \int_{B_{j}}\left(\frac{\partial}{\partial y_{1}}\right)^{2} E_{s-2} \tilde{\zeta}_{j} \tilde{u} \cdot \overline{\left(1-\Delta_{y}\right) E_{s-2} \tilde{\zeta}_{j} \tilde{u}}\left|J_{j}\right| d y+O\left(\|u\|_{s}\|u\|_{s-1}\right) \\
& =-s \int\left(\frac{\partial}{\partial y_{1}}\right)^{2} \tilde{T}_{s-2} \tilde{\zeta_{j}} \tilde{u} \cdot \overline{\Delta_{y} \tilde{T}_{s-2} \tilde{\zeta}_{j} \tilde{u}\left|J_{j}\right|} d y+O\left(\|u\|_{s}\|u\|_{s-1}\right) \\
& =-s \sum_{i=1}^{n} \int\left|\frac{\partial^{2}}{\partial y_{1} \partial y_{i}} \tilde{T}_{s-2} \tilde{\zeta}_{j} \tilde{u}\right|^{2}\left|J_{j}\right| d y+O\left(\|u\|_{s}\|u\|_{s-1}\right)
\end{aligned}
$$

where $J_{j}$ is the Jacobian of the mapping $\kappa_{j}$ and $d \sigma=\left|J_{j}\right| d y$ on $\omega_{j}$. Consequently

$$
\operatorname{Re}([h, T] \gamma u, T u) \leqq C\|u\|_{s}\|u\|_{s-1} .
$$

Now for $j$ such that $\omega_{j} \cap \Gamma_{0}=\phi$, we can immediately obtain

$$
|([h, T] \gamma u, T u)| \leqq C\left(\left\|\zeta_{j} u\right\|_{s}+\|u\|_{s-1 / 2}\right)\|u\|_{s} .
$$

Thus we can obtain the inequality similar to (2.7) by the same argument as in (2.8). Combining this inequality with (3.6), we obtain

$$
\left\|u_{\varepsilon}\right\|_{s} \leqq C\left(\|f\|_{s}^{\prime}+\left\|u_{\varepsilon}\right\|_{s-1 / 2}\right)
$$

where we wrote again $u=u_{\varepsilon}$.

To complete the proof, we have only to proceed likewise in Theorem 2.1.

3.2. Let $K$ be a continuous mapping of $\mathscr{F}_{s}^{h \alpha}$ into $\mathscr{U}_{s}^{h \alpha}(s \geqq 1 / 2)$ such that

$$
\{(\alpha S+\gamma+\beta) h+H\} K f=f, \quad f \in \mathscr{F}_{s}^{h \alpha} .
$$

This $K$ is well defined by Theorem 3.1. By the same way as in Theorem 2.2 , we can apply the Riesz-Schauder theory to the equation

$$
(1-H K) u=f
$$

and can deduce

THEOREM 3.2. (i) Let $s \geqq 1 / 2$ and $f \in H_{s}(\Gamma)$. Then the equation

$$
(\alpha S+\gamma+\beta) h u=f \quad \text { on } \Gamma
$$

admits a solution $u \in H_{s}(\Gamma)$ if and only if $f$ is orthogonal to a finite-dimensional subspace $\tilde{N}_{s}$ of $H_{s}(\Gamma)$, which has the same dimension as $N=$ $\left\{u \in H_{s}(\Gamma) ;(\alpha S+\gamma+\beta) h u=0\right\}$. (ii) Every solution $u \in H_{s}(\Gamma)(s \geqq 1 / 2)$ of (3.8) belongs to $H_{t}(\Gamma)$ if $f \in H_{t}(\Gamma)$ with $t>s$. 
Remark 3.1. The null space $N$ is independent of $s$ and is contained in $C^{\infty}(\Gamma)$.

3.3. We shall investigate the possibility of $\operatorname{dim} N=0$ in Theorem 3.2. For this purpose, we introduce auxiliary functions $q_{0}, q_{1}$ in $\Omega$, associated with the function $h$ defined in Lemma 3.1. Corresponding to Lemma 2.2, we can state

Lemma 3.3. Let $V$ be a neighborhood of $\Gamma_{0}$ and $K_{1}$ be a positive constant. Then we can find two functions $q_{0}(x)$ and $q_{1}(x)$ in $C^{\infty}(\bar{\Omega})$, satisfying for $j=0,1$,

(i ) $q_{j}(x)>0$ in $\Omega$ and $q_{j}(x)=\frac{1}{2} h(x) \alpha(x)$ on $\Gamma$,

(ii) there exist two positive constants $k$ and $d$ such that

$$
k \operatorname{dis}(x, \Gamma) \leqq q_{0}(x) \quad \text { in } \Omega_{d}=\{x \in \bar{\Omega} ; \operatorname{dis}(x, \Gamma)<d\}
$$

(iii) $)_{0}$ there exists a constant $c_{3}>0$ such that the inequality

$$
\frac{1}{2} \frac{\partial q_{0}}{\partial \nu}+\frac{1}{2} \gamma(h)+\frac{1}{2} h b+h \beta>c_{3}
$$

holds on $\Gamma$.

(iii) $\partial q_{1} / \partial \nu \geqq 0$ on $\Gamma$ and for every $u \in C^{\infty}(\Gamma)$

$$
\int_{\Gamma} \frac{1}{2} \frac{\partial q_{1}}{\partial \nu}|u|^{2} d \sigma \geqq K_{1}\|u\|_{0, \Gamma-V}
$$

For the proof, we have only to note

$$
\frac{1}{2} \gamma(h)+\frac{1}{2} h b+h \beta=\frac{1}{2} \quad \text { on } \Gamma_{0},
$$

which follows from Lemma 3.1.

Setting $q(x)=q_{0}(x)+q_{1}(x)$, we consider bilinear form

$$
\begin{aligned}
B[U, V]= & \int_{\Omega}\left(\sum_{i, j=1}^{n} a_{i j} \partial_{j} U \cdot \overline{\partial_{i}(q V)}+\sum_{i=1}^{n} b_{i} \partial_{i} U \cdot \overline{q V}+c U \cdot \overline{q V}\right) d x \\
& +\int_{\Gamma}(\gamma(h u)+h \beta u+\alpha[S, h] u) \bar{v} d \sigma
\end{aligned}
$$

with $u=\left.U\right|_{\Gamma}$ and $v=\left.V\right|_{\Gamma}$. Integrating by part, we have

$$
B[U, V]=\int_{\Omega} q A U \cdot \bar{V} d x+\int_{\Gamma}\left(h \alpha \frac{\partial U}{\partial \nu}+\alpha[S, h] u+\gamma(h u)+h \beta u\right) \bar{v} d \sigma .
$$

On the other hand, it follows from (3), Lemma A.3, Lemma 3.3 and (3.2) 
that there exist constants $c_{4}>0$ and $\lambda_{0}$ such that

$$
\begin{aligned}
\operatorname{Re} B[U, U]= & \operatorname{Re} \int_{\Omega} q\left(\sum_{i, j=1}^{n} a_{i j} \partial_{j} U \cdot \overline{\partial_{i}} \bar{U}+\sum_{i=1}^{n} b_{i} \partial_{i} U \cdot \bar{U}+c U \bar{U}\right) d x \\
& +\frac{1}{2} \int_{\Omega} \sum_{i, j=1}^{n} a_{i j} \partial_{i} q \cdot \partial_{j}(U \bar{U}) d x \\
& +\operatorname{Re} \int_{\Gamma}(\gamma(h u)+h \beta u+\alpha[S, h] u) \bar{u} d \sigma \geqq \frac{c_{0}}{2}\|p \partial U\|_{0, \Omega}^{2} \\
& +\int_{\Omega}(c-\mu)|p U|^{2} d x+\frac{1}{2} \int_{\Omega} A_{0} q \cdot|U|^{2} d x \\
& +\int_{\Gamma}\left(\frac{1}{2} \frac{\partial q_{0}}{\partial \nu}+\frac{1}{2} \gamma^{*}(h)+\gamma(h)+h \beta\right)|u|^{2} d \sigma \\
& +\int_{\Gamma} \frac{1}{2} \frac{\partial q_{1}}{\partial \nu}|u|^{2} d \sigma-\mid \int_{\Gamma} \alpha[S, h] u \cdot \bar{u} d \sigma \\
& \geqq c_{4}\|p \partial U\|_{0, \Omega}^{2}-\lambda_{0}\|p U\|_{0, \Omega}^{2}+c_{3}\|u\|_{0}^{2} \\
& +\int_{\Gamma} \frac{1}{2} \frac{\partial q_{1}}{\partial \nu}|u|^{2} d \sigma-2 K_{0} \delta\|u\|_{0}^{2}-\frac{K_{0}}{4 \delta} \max _{\Gamma}|\alpha| \cdot\|u\|_{0, \Gamma-V_{\delta}}^{2} .
\end{aligned}
$$

Taking, in Lemma 3.3, $V=V_{\delta}$ with $\delta=c_{3} / 4 K_{0}$ and $K_{1}=K_{0} \max _{\Gamma}|\alpha| / 4 \delta$, we obtain, for all $U \in C^{\infty}(\bar{\Omega})$,

$$
\operatorname{Re} B[U, U] \geqq c_{4}\|p \partial U\|_{0, \Omega}^{2}-\lambda_{0}\|p U\|_{0, \Omega}^{2}+\frac{c_{3}}{2}\|u\|_{0}^{2} .
$$

ThEOREM 3.3. Let $N(\lambda)=\left\{u \in H_{s}(\Gamma)(s \geqq 1 / 2) ;\left(\alpha S_{\lambda}+\gamma+\beta\right) h u=0\right\}$. Then $\operatorname{dim} N(\lambda)=0$ for all $\lambda \geqq \lambda_{0}$.

Proof. Let $u \in N(\lambda)$. By the same argument as in Theorem 2.3, we can first establish $u \in C^{\infty}(\Gamma)$. The solution $U$ of the Dirichlet problem, $A_{\lambda} U=0$ in $\Omega$ and $U=u$ on $\Gamma$, is in $C^{\infty}(\bar{\Omega})$ and satisfies $h \alpha \partial U / \partial \nu+\gamma(h u)$ $+h \beta u+\alpha\left[S_{\lambda}, h\right] u=0$, because $h \alpha S_{\lambda} u+\gamma(h u)+h \beta u+\alpha\left[S_{\lambda}, h\right] u=0$ on $\Gamma$. Therefore, by (3.9) we have

$$
B[U, V]+\lambda \int_{\Omega} q U \bar{V} d x=0
$$

for all $V \in C^{\infty}(\Omega)$. Applying (3.11) to this $U$, we have $u=0$.

Q.E.D.

3.4. We return again to the problem (1)-(2). We shall say a function $U$ contained in $H_{s+1}(\Omega)$ to vanish on $\Gamma_{0}$, if there exists $u_{1} \in H_{s+1 / 2}(\Gamma)$ such that $U=h u_{1}$ on $\Gamma$. In that case we write briefly $U=0$ on $\Gamma_{0}$.

TheOREM 3.4. (i) Let $s \geqq 0$ and $(F, f)$ belong to $H_{s}(\Omega) \times H_{s+1 / 2}(\Gamma)$. 
Then the problem

$$
\left\{\begin{array}{l}
A U=F \quad \text { in } \Omega \\
\alpha \frac{\partial U}{\partial \nu}+\gamma U+\beta U=f \quad \text { on } \Gamma \\
U=0 \quad \text { on } \Gamma_{0}
\end{array}\right.
$$

admits a solution $U \in H_{s+1}(\Omega)$ if and only if $(F, f)$ is orthogonal to a finite dimensional subspace of $H_{s}(\Omega) \times H_{s+1 / 2}(\Gamma)$, and the space of solutions of (3.12) with $F=f=0$ has the finite dimension. (ii) If $(F, f) \in H_{t}(\Omega) \times$ $H_{t+1 / 2}(T)(t>s \geqq 0)$, every solution $U \in H_{s+1}(\Omega)$ of $(3.12)$ belongs to $H_{t+1}(\Omega)$.

The proof is established by similar argument as in the proof of Theorem 2.4.

Now we can state a uniqueness theorem as a corollary of Theorems 3.3 and 3.4 .

THEOREM 3.5. Let $\lambda_{0}$ be the number appearing in (3.11) and $\lambda$ be any number such that $\lambda \geqq \lambda_{0}$. Then for every $(F, f) \in H_{s}(\Omega) \times H_{s+1 / 2}(\Gamma)(s \geqq 0)$, we can find one and only one $U \in H_{s+1}(\Omega)$ satisfying

$$
\left\{\begin{array}{l}
(A+\lambda) U=F \quad \text { in } \Omega \\
\alpha \frac{\partial U}{\partial \nu}+\gamma U+\beta U=f \quad \text { on } \Gamma \\
U=0 \quad \text { on } \Gamma_{0} .
\end{array}\right.
$$

Moreover the inequality

$$
\|U\|_{s+1, \Omega} \leqq C_{s}\left(\|F\|_{s, \Omega}+\|f\|_{s+1 / 2}\right)
$$

holds with a suitable constant $C_{s}>0$.

\section{Chapter 4. The case of type (III)}

4.0. In this final chapter, we consider the manifold $\Gamma_{0}$ of type (III). Suppose that $\Gamma_{0}$ is a closed manifold which devides $\Gamma$ into two open sets $\Gamma_{-}, \Gamma_{+}$so that $\alpha<0$ on $\Gamma_{-}, \alpha>0$ on $\Gamma_{+}$and $\alpha=0$ on $\Gamma_{0}$, and that the tangential vector field $-\gamma$ is transversal from $\Gamma_{+}$to $\Gamma_{-}$on $\Gamma_{0}$. So that we can assume that $\alpha, \gamma$ and $\beta$ are the same things as in $\S 3$. Then we must treat the boundary condition

$$
\alpha \frac{\partial U}{\partial \nu}-\gamma U+\beta U=f \quad \text { on } \Gamma
$$


that is, solve the equation

$$
(\alpha S-\gamma+\beta) u=f \quad \text { on } \Gamma .
$$

To do so we consider the equation

$$
h(\alpha S-\gamma+\beta) u=f
$$

instead of it, with the same function $h$ as the one defined in Lemma 3.1, and shall give a solution $u$ in $H_{0}(\Gamma)$ which is smooth in $\Gamma \backslash \Gamma_{0}$.

4.1. We first study the equation (4.1) and set

$$
Q[u, v]=(h(\alpha S-\gamma+\beta) u, v) .
$$

a simple calculation gives

$$
\text { (4.2) } \begin{aligned}
\operatorname{Re} Q[u, u]= & (h \alpha \theta u, \theta u)+\left(\left(\frac{[[h \alpha, \theta], \theta]}{2}+\frac{\left[h \alpha, S-S^{*}\right]}{4}-h \alpha M\right) u, u\right) \\
& +\left(\left(\frac{1}{2} \gamma(h)-\frac{1}{2} h b+h \beta\right) u, u\right) .
\end{aligned}
$$

Since $\gamma(h)=1$ near $\Gamma_{0}$ (see Lemma 3.1), there exists a constant $R>0$ such that

$$
R h \alpha+\frac{1}{2} \gamma(h)+h \beta-\frac{1}{2} b h>0 \quad \text { on } \Gamma .
$$

Let $H$ be a pseudo-differential operator on $\Gamma$ defined by

$$
H=R h \alpha+h \alpha M-\frac{[[h \alpha, \theta], \theta]}{2}-\frac{\left[h \alpha, S-S^{*}\right]}{4}
$$

and set, for $\varepsilon$ such that $0<\varepsilon \leqq 1$,

$$
Q_{\varepsilon}[u, v]=Q[u, v]+(H u, v)+\varepsilon((S+M) u, v) .
$$

It then follows from (4.2) and (4.3) that there exists a constant $c_{2}>0$ such that

$$
\operatorname{Re} Q_{\varepsilon}[u, u] \geqq c_{2}\|u\|^{2}+\varepsilon\|\theta u\|_{0}^{2} .
$$

Accordingly, for every $f \in C^{\infty}(\Gamma)$ we can find $u_{\varepsilon} \in C^{\infty}(\Gamma)$ satisfying

$$
\{h(\alpha S-\gamma+\beta)+H+\varepsilon(S+M)\} u_{\varepsilon}=f \quad \text { on } \Gamma
$$

and

$$
\left\|u_{\varepsilon}\right\|\left|\leqq C_{0}\|f \mid\|^{\prime}\right.
$$


with a constant $C_{0}>0$ not depending on $\varepsilon$.

We now introduce sequences $\hat{\mathscr{U}}_{m / 2}$ and $\hat{\mathscr{F}}_{m / 2}(m=0,1,2, \cdots)$ of subspaces of $\mathscr{U}^{h \alpha}$ and $\mathscr{F}^{h \alpha}$, respectively; $\hat{\mathscr{U}}_{m / 2}$ and $\hat{\mathscr{F}}_{m / 2}$ are the Hilbert spaces obtained by the completion of $C^{\infty}(\Gamma)$ with respect to the norms

$$
\langle u\rangle_{m / 2}=\left(\sum_{k=0}^{m}\left\|h^{k} u\right\|_{k / 2}^{2}\right)^{1 / 2}
$$

and

$$
\langle f\rangle_{m / 2}^{\prime}=\left(\sum_{k=0}^{m}\left\|h^{k} f\right\|_{k / 2}^{\prime 2}\right)^{1 / 2},
$$

respectively. It is easily seen that

$$
\mathscr{U}^{h \alpha}=\hat{\mathscr{U}}_{0} \supset \hat{\mathscr{U}}_{1 / 2} \supset \cdots, \quad \mathscr{F}^{h \alpha}=\hat{\mathscr{F}}_{0} \supset \hat{\mathscr{F}}_{1 / 2} \supset \cdots
$$

with continuous injections. Then we can state

TheOREM 4.1. Let $m$ be a non-nagative integer. For every $f \in \hat{\mathscr{F}}_{m / 2}$, we can find one and only one $u \in \hat{\mathscr{U}}_{m / 2}$ satisfying the equation

$$
\{h(\alpha S-\gamma+\beta)+H\} u=f \quad \text { on } \Gamma \text {. }
$$

and the inequality

$$
\langle u\rangle_{m / 2} \leqq C_{m / 2}\left\langle\langle f\rangle_{m / 2}^{\prime}\right.
$$

with a constant $C_{m / 2}>0$ independent of $f$. Moreover the $u$ is unique in $H_{0}(\Gamma)$.

Proof. Suppose first $f \in C^{\infty}(\Gamma)$ and substitute $u=T_{s}^{(j)}\left(h^{m} u_{s}\right)(s=\mathrm{m} / 2)$ in (4.4). Writting, for simplicity, $T_{s}^{(j)}$ as $T$ and $u_{s}$ as $u$, we have, for $j=1, \cdots, \ell$,

$$
\begin{aligned}
c_{2}\left\|T h^{m} u\right\|^{2}+\varepsilon\left\|\theta T h^{m} u\right\|_{0}^{2} \leqq \operatorname{Re} Q_{\varepsilon}\left[T h^{m} u, T h^{m} u\right] \\
=\operatorname{Re}\left(\{h(\alpha S-\gamma+\beta)+H+\varepsilon(S+M)\} T h^{m} u, T h^{m} u\right) \\
=\operatorname{Re}\left(T h^{m}\{\quad\} u, T h^{m} u\right)+\operatorname{Re}\left(\left[\{\quad\}, T h^{m}\right] u, T h^{m} u\right) \\
=\operatorname{Re}\left(T h^{m} f, T h^{m} u\right)+\operatorname{Re}\left(\left[h \beta+H, T h^{m}\right] u, T h^{m} u\right) \\
\quad+\operatorname{Re}\left(\left[h \alpha S-h \gamma+\varepsilon S, T h^{m}\right] u, T h^{m} u\right)=\mathrm{I}+\mathrm{II}+\mathrm{III} .
\end{aligned}
$$

First we shall estimate the second term II of the right hand side which is written as $\operatorname{Re} A_{j}$. Rewritting as

$$
\begin{aligned}
{\left[h \beta+H, T h^{m}\right] } & =[h \beta+H, T] h^{m}+T\left[H, h^{m}\right] \\
& =[h \beta+H, T] h^{m}+T \sum_{k=0}^{m-1} H_{k-m} h^{k},
\end{aligned}
$$


we have

$$
\operatorname{Re} A_{j}=\operatorname{Re}\left(\theta\left[h \beta+H, T h^{m}\right] u, \theta^{-1} T h^{m} u\right) \leqq C\left\langle\langle u\rangle_{s-1 / 2}^{2},\right.
$$

where $H_{k-m}$ is a pseudo-differential operator of order $k-m$ and, as well as in the following, $C, C_{0}, C_{1}, \cdots$ denote positive constants not depending on $\varepsilon$.

Now we shall estimate the third term III. To do so we represent this term as the sum of $\operatorname{Re} X_{j}, \operatorname{Re} Y_{j}$ and $\operatorname{Re} Z_{j}$, where

$$
\begin{aligned}
X_{j} & =\left(\left[h \alpha S, T h^{m}\right] u, T h^{m} u\right), \\
Y_{j} & =\left(\left[\varepsilon S, T h^{m}\right] u, T h^{m} u\right), \\
Z_{j} & =-\left(\left[h \gamma, T h^{m}\right] u, T h^{m} u\right) .
\end{aligned}
$$

Since

$$
X_{j}=\left(T h \alpha\left[S, h^{m}\right] u, T h^{m} u\right)+\left([h \alpha S, T] h^{m} u, T h^{m} u\right)
$$

and the second term has the same form as $X_{j}$ in $\S 3$, we have only calculate the first. Noting that

$$
\left[S, h^{m}\right]=\sum_{k=0}^{m-1} S_{1+k-m} h^{k},
$$

with pseudo-differential operators $S_{1+k-m}$ of order $1+k-m$, we obtain

$\operatorname{Re}\left(T h \alpha\left[S, h^{m}\right] u, T h^{m} u\right)$

$$
\begin{aligned}
& \leqq \operatorname{Re} \sum_{k=0}^{m-1}\left\{\left(T S_{1+k-m} h^{k} u, h \alpha T h^{m} u\right)+\left(\theta[T, h \alpha] S_{1+k-m} h^{k} u, \theta^{-1} T h^{m} u\right)\right\} \\
& =\operatorname{Re} \sum_{k=0}^{m-1}\left(\theta^{-1} T S_{1+k-m} h^{k} u, h \alpha \theta T h^{m} u\right)+O\left(\langle u\rangle_{s-1 / 2}^{2}\right) \\
& \leqq C\left(\sum_{k=0}^{m-1}\left\|h^{k} u\right\|_{k / 2}\left\|h \alpha \theta T h^{m} u\right\|_{0}+\left\langle\langle u\rangle_{s-1 / 2}^{2}\right) .\right.
\end{aligned}
$$

This and (3.5) show that for every $\delta$ there exists a constant $C_{\delta}>0$ such that

$$
\operatorname{Re} X_{j} \leqq \delta\left\|\sqrt{h \alpha} \theta T h^{m} u\right\|_{0}^{2}+C_{\hat{o}}\langle u\rangle_{s-1 / 2}^{2} .
$$

By the same argument as in the above, we can obtain, for every $\delta>0$,

$$
\operatorname{Re} Y_{j} \leqq \varepsilon\left(\delta\left\|\theta T h^{m} u\right\|_{0}^{2}+C_{\delta}\langle u\rangle_{s-1 / 2}^{2}\right)
$$

with a constant $C_{\delta}>0$.

Finally we consider $Z_{j}$ which is written as 


$$
\begin{aligned}
Z_{j}= & -\left([h \gamma, T] h^{m} u, T h^{m} u\right)-\left(T h\left[\gamma, h^{m}\right] u, T h^{m} u\right) \\
= & -\left([h, T] \gamma h^{m} u, T h^{m} u\right)-\left(h[\gamma, T] h^{m} u, T h^{m} u\right) \\
& -m\left(T h^{m} u, T h^{m} u\right)-m\left(T(\gamma(h)-1) h^{m} u, T h^{m} u\right) .
\end{aligned}
$$

Consequently

$$
\begin{aligned}
\operatorname{Re} Z_{j} \leqq & -\operatorname{Re}\left([h, T] \gamma h^{m} u, T h^{m} u\right)-m\left\|T h^{m} u\right\|_{0}^{2} \\
& +\mid\left(h[\gamma, T] h^{m} u, T h^{m} u\right)+C\left\|(\gamma(h)-1) h^{m} u\right\|_{s}\left\|h^{m} u\right\|_{s} .
\end{aligned}
$$

It then follows from (3.5) and the fact that $\gamma(h)=1$ near $\Gamma_{0}$ that for every $\delta>0$ there exists a constant $C_{\delta}>0$ such that

$$
\begin{aligned}
\operatorname{Re} Z_{j} \leqq & -\operatorname{Re}\left([h, T] \gamma h^{m} u, T h^{m} u\right)-m\left\|T h^{m} u\right\|_{0}^{2} \\
& +\delta\left\|h^{m} u\right\|_{s}^{2}+C_{\delta}\left\|h^{m} u\right\|_{s-1 / 2}^{2} .
\end{aligned}
$$

By (3.7) we have, for $j$ such that $\omega_{j} \cap \Gamma_{0} \neq \phi$,

$-\operatorname{Re}\left(\left[h, T_{s}^{(j)}\right] \gamma h^{m} u, T_{s}^{(j)} h^{m} u\right)$

$$
\begin{aligned}
= & -s \int_{B_{j}}\left(\frac{\partial}{\partial y_{1}}\right)^{2} E_{s-2}\left(\tilde{\zeta}_{j} y_{1}^{m} \tilde{u}\right) \cdot \overline{\left(1-\Delta_{y}\right) E_{s-2}\left(\zeta_{j} y_{1}^{m} \tilde{u}\right)}\left|J_{j}\right| d y \\
& +O\left(\left\|h^{m} u\right\|_{s}\left\|h^{m} u\right\|_{s-1}\right) \\
= & s \int_{B_{j}}\left(\frac{\partial}{\partial y_{1}}\right)^{2} E_{s-2}\left(\tilde{\zeta}_{j} y_{1}^{m} \tilde{u}\right) \cdot \overline{\Delta_{y} E_{s-2}\left(\tilde{\zeta}_{j} y_{1}^{m} \tilde{u}\right)}\left|J_{j}\right| d y \\
& +O\left(\left\|h^{m} u\right\|_{s}\left\|h^{m} u\right\|_{s-1}\right) \\
= & s \int\left|\Delta_{y} \tilde{T}_{s-2}\left(\tilde{\zeta}_{j} y_{1}^{m} \tilde{u}\right)\right|^{2}\left|J_{j}\right| d y-s \sum_{k=2}^{n} \int\left(\frac{\partial}{\partial y_{k}}\right)^{2} \tilde{T}_{s-2}\left(\tilde{\zeta}_{j} y_{1}^{m} \tilde{u}\right) \\
& \frac{\cdot \Delta_{y} \tilde{T}_{s-2}\left(\tilde{\zeta}_{j} y_{1} \tilde{u}\right)\left|J_{j}\right| d y+O\left(\left\|h^{m} u\right\|_{s}\left\|h^{m} u\right\|_{s-1}\right)}{=} \\
& s \int\left|\Delta_{y} \tilde{T}_{s-2}\left(\tilde{\zeta}_{j} y_{1}^{m} \tilde{u}\right)\right|^{2}\left|J_{j}\right| d y-s \sum_{k=2}^{n} \sum_{i=1}^{n} \int\left|\frac{\partial^{2}}{\partial y_{k} \partial y_{1}} \tilde{T}_{s-2}\left(\tilde{\zeta}_{j} y_{1}^{m} \tilde{u}\right)\right|^{2}\left|J_{j}\right| d y \\
& +O\left(\left\|h^{m} u\right\|_{s}\left\|h^{m} u\right\|_{s-1}\right) .
\end{aligned}
$$

Since

$$
\left\|T_{s}^{(j)} h^{m} u\right\|_{0}^{2}=\left.\int \Delta_{y} \tilde{T}_{s-2} \tilde{\zeta}_{j} y_{1}^{m} \tilde{u}\right|^{2}\left|J_{j}\right| d y+O\left(\left\|h^{m} u\right\|_{s}\left\|h^{m} u\right\|_{s-1}\right)
$$

we have by (4.9)

$$
\begin{aligned}
\operatorname{Re} Z_{j} \leqq & \left.(s-m) \int \Delta_{y} \tilde{T}_{s-2}\left(\tilde{\zeta}_{j} y_{1}^{m} \tilde{u}\right)\right|^{2}\left|J_{j}\right| d y+C\left\|h^{m} u\right\|_{s}\left\|h^{m} u\right\|_{s-1} \\
& +\delta\|\| h^{m} u\left\|_{s}^{2}+C_{\delta}\right\| h^{m} u \|_{s-1 / 2}^{2} .
\end{aligned}
$$

Accordingly, for any $\delta>0$ there exists another constant $C_{\delta}>0$ such that 


$$
\operatorname{Re} Z_{j} \leqq \delta\left\|h^{m} u\right\|_{s}^{2}+C_{\delta}\left\|h^{m} u\right\|_{s-1 / 2}^{2}
$$

by taking account of $s-m=-m / 2$. This remain valid also for $j$ such that $\omega_{j} \cap \Gamma_{0}=\phi$. Thus it follows from (4.6), (4.7), (4.8) and (4.10) that

$$
\left\langle\left\langle u_{\varepsilon}\right\rangle_{s}^{2} \leqq C_{s}\left(\langle f\rangle_{s}^{\prime 2}+\left\langle\left\langle u_{s}\right\rangle_{s-1 / 2}^{2}\right)\right.\right.
$$

is valid for all $s=m / 2(m=0,1,2, \cdots)$. By (4.5) and induction on $m$, we obtain

$$
\left\langle\left\langle u_{e}\right\rangle_{s} \leqq C_{s}\left\langle\langle f\rangle_{s}^{\prime},\right.\right.
$$

for all $s=m / 2(m=0,1,2, \cdots)$, with a constant $C_{s}>0$ not depending on $\varepsilon$.

Let $m$ be fixed. Then it follows from the theorem of Banach-Sacks that there exists a decreasing sequence $\varepsilon_{1}, \varepsilon_{2}, \cdots$ converging to zero such that

$$
v_{n}=\frac{u_{s_{1}}+\cdots+u_{\varepsilon_{n}}}{n}
$$

converges to some $u$ in $\hat{\mathscr{U}}_{s}(s=m / 2)$. Accordingly the $u$ satisfies

$$
\left\{\begin{array}{l}
\{h(\alpha S-\gamma+\beta)+H\} u=f \quad \text { on } \Gamma \\
\langle u\rangle_{s} \leqq C_{m}\langle f\rangle_{s}^{\prime} .
\end{array}\right.
$$

Now let $f \in \hat{\mathscr{F}}_{s}$ and choose $f_{j}$ in $C^{\infty}(\Gamma)$ so that $f_{j} \rightarrow f$ in $\hat{\mathscr{F}}_{s}$ as $j \rightarrow \infty$. For each $f_{j}$, we can find $u_{j}$ in $\hat{\mathscr{U}}_{s}$ satisfying (4.11) with $f=f_{j}$. As is easily seen, $u_{j}$ converges in $\hat{\mathscr{U}}_{s}$ as $j \rightarrow \infty$ and the limit $u$ satisfies (4.11).

Finally we shall prove the uniqueness of $u$ in $H_{0}(\Gamma)$. To do so, we consider the dual problem

$$
\left\{(\alpha S-\gamma+\beta)^{*} h+H\right\} v=g .
$$

Let $g$ be in $C^{\infty}(\Gamma)$. Then, by (4.4), we can find $v_{\varepsilon} \in C^{\infty}(\Gamma)$ such that

$$
\left\{(\alpha S-\gamma+\beta)^{*} h+H+\varepsilon\left(S^{*}+M\right)\right\} v_{\varepsilon}=g \quad \text { on } \Gamma
$$

and $\left\|v_{s}\right\| \leqq C_{0}\|g\|^{\prime}$ with a constant $C_{0}$ independent of $\varepsilon$. Substitute $u=$ $T_{s}^{(j)} v_{s}(s$ real $\geqq 1 / 2)$ in (4.4). Then following the same argument as in the proof of Theorem 3.1, we can derive the inequality

$$
\left\|v_{s}\right\|_{s} \leqq C_{s}\|g\|_{s}^{\prime} .
$$

Thus we can prove that for every $g \in \mathscr{F}_{s}^{h \alpha}$ there exists one and only one 
$v \in \mathscr{U}_{s}^{h \alpha}$ satisfying (4.12). Furthermore the result of Theorem 3.2 remains valid for the equation $(\alpha S-\gamma+\beta)^{*} h v=g$.

Now let $u$ be in $H_{0}(\Gamma)$ and satisfy $\{h(\alpha S-\gamma+\beta)+H\} u=0$ on $\Gamma$. This means that

$$
\left(u,\left\{(\alpha S-\gamma+\beta)^{*} h+H\right\} v\right)=0
$$

for all $v \in C^{\infty}(\Gamma)$. Hence we have $(u, g)=0$ for all $g \in C^{\infty}(\Gamma)$. Thus the proof of Theorem 4.1 is completed.

4.2. Let $m$ be a non-negative integer. By $\mathscr{H}_{s}(\Gamma)(s=m / 2)$ we denote the Hilbert space obtained by the completion of $C^{\infty}(\Gamma)$ with respect to the norm

$$
\langle u\rangle_{s}=\left(\sum_{k=0}^{m}\left\|h^{k} u\right\|_{k / 2}^{2}\right)^{1 / 2}
$$

It easily follows from Proposition 1.3 that for all $s=m / 2$

$$
\hat{\mathscr{F}}_{s} \supset \mathscr{H}_{s}(\Gamma) \supset \hat{\mathscr{U}}_{s}
$$

is valid and the injections are continuous. Using Theorem 4.1, we can define a continuous mapping $K$ of $\hat{\mathscr{F}}_{s}$ into $\hat{\mathscr{U}}_{s}$ such that

$$
\{h(\alpha S-\gamma+\beta)+H\} K=1 .
$$

Hence (4.13) guarantees that $K$ is also a continuous mapping of $\mathscr{H}_{s}(\Gamma)$ into itself. Thus we have only to consider the equation

$$
(1-H K) g=f
$$

in order to solve the original equation

$$
h(\alpha S-\gamma+\beta) u=f .
$$

THEOREM 4.2. (i) Let $m$ be a non-negative integer and $f$ be in $\mathscr{H}_{s}(\Gamma)$ $(s=m / 2)$. Then the equation (4.14) admits a solution $u \in \mathscr{H}_{s}(\Gamma)$ if and only if $f$ is orthogonal to a finite-dimensional subspace $\hat{N}_{s}$ of $\mathscr{H}_{s}(\Gamma)$ which has the same dimension as $N=\left\{u \in \mathscr{H}_{s}(\Gamma) ; h(\alpha S-\gamma+\beta) u=0\right\}$. (ii) Every solution $u$ in $\mathscr{H}_{s}(\Gamma)(s=m / 2)$ of (4.14) belongs to $\mathscr{H}_{t}(\Gamma)$ if $f \in \mathscr{H}_{t}(\Gamma)(t=$ $\ell / 2)$ with integer $\ell>m$.

Proof. In order to show (i), it is sufficient to prove the compactness of the operator $H K$ on $\mathscr{H}_{s}(\Gamma)$, where $s=m / 2$. If $g \in \mathscr{H}_{s}(\Gamma)$, then $K g \in \hat{\mathscr{U}}_{s}$, that is, $h^{k} K g \in \mathscr{U}_{k / 2}^{h \alpha}$ for $k=0,1, \cdots, m$. Hence we have 


$$
h^{k} H K g=H h^{k} K g+\left[h^{k}, H\right] K g=H h^{k} K g-\sum_{i=0}^{k-1} H_{i-k} h^{i} K g,
$$

where $H_{i-k}$ is a pseudo-differential operator of order $i-k$. Proposition 1.4 implies $h^{k} H K g \in H_{(k+1) / 2}(\Gamma)$ for $k=0,1, \cdots, m$, from which it follows that $H K$ is a compact operator on $\mathscr{H}_{s}(\Gamma)$.

(ii) If $u$ is in $\mathscr{H}_{s}(\Gamma)(s=m / 2)$ and satisfy (4.14), then $H(\alpha S-\gamma+\beta) u$ $+H u=f+H u$. By Proposition 1.5, we have $H u \in \hat{\mathscr{F}}_{s+1 / 2}$, since $h^{k} u \in$ $H_{k / 2}(\Gamma)$ for $k=0,1, \cdots, m$ and

$$
h^{k} H u=H h^{k} u-\sum_{i=0}^{k-1} H_{i-k} h^{i} u .
$$

Therefore $u=K f+K H u \in \mathscr{H}_{t}(\Gamma)$ if $\ell \leqq m+1$. If $\ell>m+1, u \in \mathscr{H}_{s+1 / 2}(\Gamma)$. After repeating this argument, we obtain $u \in \mathscr{H}_{\ell / 2}(\Gamma)$.

Q.E.D.

Remark 4.1. The null space $N$ is independent of $s$ and is contained in $\bigcap_{m-1}^{\infty} \mathscr{H}_{m / 2}(\Gamma)$, which easily follows from Theorem 4.2 (ii).

4.3. We shall again study the possibility of $\operatorname{dim} N=0$ in Theorem 4.2. To do so we first introduce a $C^{\infty}$-function $q(x)$ in $\Omega$ in like manner as in Lemmas 2.2 and 3.2.

LEMMA 4.2. We can find a function $q(x)$ in $C^{\infty}(\Omega)$ satisfying (i) and (ii) of Lemma 2.2, and

(iii) the inequality

$$
\frac{1}{2} \frac{\partial q}{\partial \nu}+\frac{1}{2} \gamma(h)-\frac{1}{2} h b+h \beta>c_{3}
$$

holds on $\Gamma$ with a constant $c_{3}>0$, where $h$ is the function introduced in Lemma 3.1.

Now we consider a bilinear form

$$
\begin{aligned}
B[U, V]= & \int_{\Omega}\left(\sum_{i, j=1}^{n} a_{i j} \partial_{j} U \cdot \overline{\partial_{i}(q V)}+\sum_{i=1}^{n} b_{i} \partial_{i} U \cdot \overline{q V}+c U \cdot \overline{q V}\right) d x \\
& +\int_{\Gamma}(-h \gamma(u)+h \beta u) \bar{v} d \sigma
\end{aligned}
$$

$u$ and $v$ being the restrictions of $U$ and $V$ on $\Gamma$, respectively. Similar calculation as in (2.14) and (2.15) leads to

$$
B[U, V]=\int_{\Omega} q A U \cdot \bar{V} d x+\int_{\Gamma}\left(h \alpha \frac{\partial U}{\partial \nu}-h \gamma(u)+h \beta u\right) \bar{v} d \sigma
$$


and

$$
\operatorname{Re} B[U, V] \geqq c_{4}\|p \partial U\|_{0, \Omega}^{2}-\lambda_{0}\|p U\|_{0, \Omega}^{2}+c_{3}\|u\|_{0}^{2}
$$

for all $U$ and $V$ in $C^{\infty}(\bar{\Omega})$, where $c_{4}$ is a positive constant, $p=\sqrt{q}$ and $\lambda_{0}$ is a real number.

TheOREM 4.3. Let $N(\lambda)=\left\{u \in \mathscr{H}_{m / 2}(\Gamma)\right.$ (m integer $\left.\geqq 0\right) ; h\left(\alpha S_{\lambda}-\gamma+\beta\right) u$ $=0\}$. Then $\operatorname{dim} N(\lambda)=0$ for all $\lambda \geqq \lambda_{0}$.

Proof. First we prove that if $v$ is in $H_{s}(\Gamma)(s \geqq 1 / 2)$ and satisfies $\left(\alpha S_{\lambda}-\lambda+\beta\right)^{*} h v=0$ with $\lambda \geqq \lambda_{0}$, then $v=0$. It is obvious that $v \in C^{\infty}(\Gamma)$ (see the final part of Proof of Theorem 4.1). Let $V$ be in $C^{\infty}(\bar{\Omega})$, and satisfy $A_{2} V=0$ in $\Omega$ and $V=v$ on $\Gamma$. It then follows from (4.15) that for every $U \in C^{\infty}(\bar{\Omega})$ such that $A_{\lambda} U=0$ in $\Omega$

$$
\begin{aligned}
B[U, V]+\lambda \int_{\Omega} q U \cdot \bar{V} d x & =\left(h\left(\alpha S_{\lambda}-\gamma+\beta\right) u, v\right) \\
& =\left(u,\left(\alpha S_{\lambda}-\gamma+\beta\right)^{*} h v\right)=0,
\end{aligned}
$$

where $u=\left.U\right|_{\Gamma}$. Taking $V$ as $U$ in (4.17) and applying (4.16) for $U=V$, we have $v=0$. Accordingly, for every $g \in C^{\infty}(\Gamma)$, we can find $v \in C^{\infty}(\Gamma)$ so that $\left(\alpha S_{\lambda}-\gamma+\beta\right)^{*} h v=g$ (cf. Theorem 3.2). Now let $u \in N(\lambda)$. We then have

$$
0=\left(u,\left(\alpha S_{\lambda}-\gamma+\beta\right)^{*} h v\right)=(u, g)
$$

Hence $u=0$.

4.4. Let $\eta(x)$ be a $C^{\infty}$-function in $\Omega_{1}$ such that $\eta(x)=h(x)^{2}$ on $\Gamma$ and $\eta(x)>0$ in $\Omega$. For every non-negative integer $m$ and real number $\mu$, we denote by $\mathscr{H}_{m, \mu}(\Omega)$ the Hilbert space obtained by the completion of $C^{\infty}(\bar{\Omega})$ with respect to the norm

$$
\|U\|_{m, \mu, \Omega}=\left(\sum_{k=0}^{m}\left\|\eta^{k} U\right\|_{k+\mu, \Omega}^{2}\right)^{1 / 2} .
$$

Then it is easily seen that if $U \in \mathscr{H}_{m, \mu}(\Omega)$, then $\partial_{j} U \in \mathscr{H}_{m, \mu-1}(\Omega)$ for $j=1$, $\cdots, n$. Moreover we can prove that if $\mu>1 / 2$, then the restriction on $\Gamma$ of $U \in \mathscr{H}_{m, \mu}(\Omega)$ is in $\mathscr{H}_{m}(\Gamma)$, by using the following

Proposition 4.1. Let $k$ be an integer $\geqq 1$. Then there exists a constant $C_{k}>0$ such that the inequality

$$
\|h u\|_{k-1 / 2} \leqq C_{k}\left(\|u\|_{k-1}+\left\|h^{2} u\right\|_{k}\right)
$$


holds for every $u \in C^{\infty}(\Gamma)$.

Proof. It is sufficient to prove (4.18) when $u$ has its support in $\omega_{j}$ such that $\omega_{j} \cap \Gamma_{0} \neq \phi$. Then, by the transformation $y=\kappa_{j}(x)$, the inequality (4.18) is altered to

$$
\left\|y_{1} \phi\right\|_{k-1 / 2, R^{n-1}} \leqq C_{k}\left(\|\phi\|_{k-1, R^{n-1}}+\left\|y_{1}^{2} \phi\right\|_{k, R^{n-1}}\right),
$$

where $\phi(y)=u\left(\kappa_{j}^{-1}(y)\right)$. Integrating by part, we have

$$
\begin{aligned}
\left\|y_{1} \phi\right\|_{k-1 / 2, R^{n-1}}^{2} & =\int\left(1+|\xi|^{2}\right)^{k-1 / 2} \frac{\partial \hat{\phi}}{\partial \xi_{1}} \frac{\partial \hat{\phi}}{\partial \xi} d \xi \\
& =-\int\left\{(2 k-1) \xi_{1}\left(1+|\xi|^{2}\right)^{k-3 / 2} \frac{\partial \hat{\phi}}{\partial \xi_{1}}+\left(1+|\xi|^{2}\right)^{k-1 / 2} \frac{\partial^{2} \hat{\phi}}{\partial \xi_{1}^{2}}\right\} \hat{\phi} d \xi \\
& \leqq(2 k-1) \int\left(1+|\xi|^{2}\right)^{k-1}|\hat{\phi}|\left|\frac{\partial \hat{\phi}}{\partial \xi_{1}}\right| d \xi \\
& +\int\left(1+|\xi|^{2}\right)^{k-1 / 2}|\hat{\phi}|\left|\frac{\partial^{2} \hat{\phi}}{\partial \xi_{1}^{2}}\right| d \xi .
\end{aligned}
$$

Accordingly, by the Schwarz inequality,

$$
\left\|y_{1} \phi\right\|_{k-1 / 2}^{2} \leqq(2 k-1)\|\phi\|_{k-1}\left\|y_{1} \phi\right\|_{k-1}+\|\phi\|_{k-1}\left\|y_{1}^{2} \phi\right\|_{k},
$$

where we omit the suffix $R^{n-1}$. This immediately implies (4.19), and hence (4.18) is proved.

Now we can state

THEOREM 4.4. (i) Let $m$ be a non-negative integer and $(F, f)$ belong to $\mathscr{H}_{m, 0}(\Omega) \times \mathscr{H}_{m}(\Gamma)$. Then the problem

$$
\left\{\begin{array}{l}
A U=F \quad \text { in } \Omega \\
h\left(\alpha \frac{\partial U}{\partial \nu}-\gamma U+\beta U\right)=h f \quad \text { on } \Gamma
\end{array}\right.
$$

admits a solution $U$ in $\mathscr{H}_{m, 1 / 2}(\Omega)$ if and only if $(F, f)$ is orthogonal to a finite-dimensional subspace of $\mathscr{H}_{m, 0}(\Omega) \times \mathscr{H}_{m}(\Gamma)$ and the space of solutions in $\mathscr{H}_{m, 1 / 2}(\Omega)$ of (4.20) with $F=f=0$ has the finite dimension. (ii) If $(F, f)$ belongs to $\mathscr{H}_{\ell, 0}(\Omega) \times \mathscr{H}_{\ell}(\Gamma)$ ( $\ell$ integer $>m$ ), then $U \in \mathscr{H}_{\ell, 1 / 2}(\Omega)$.

Proof (cf. Proof of Theorem 2.4). (i) Let $(F, f)$ be in $\mathscr{H}_{m, 0}(\Omega) \times \mathscr{H}_{m}(\Gamma)$. By $V$ we mean a solution in $H_{2}(\Omega)$ of the Dirichlet problem; $A V=F$ in $\Omega$ and $V=0$ an $\Gamma$. Since we have

$$
A\left(\eta^{k} V\right)=\eta^{k} F+\left[A, \eta^{k}\right] V=\eta^{k} F+P_{1} \eta^{k-1} V+P_{0} \eta^{k-2} V
$$


for $k \geqq 1\left(P_{0}=0\right.$ if $\left.k=1\right)$, with some differential operators $P_{i}(i=0,1)$ of order $i$, it then follows from (2.17) that

$$
\left\|\eta^{k} V\right\|_{k+2} \leqq C\left(\left\|\eta^{k} F\right\|_{k}+\left\|\eta^{k-1} V\right\|_{k-1+2}+\left\|\eta^{k-2} V\right\|_{k-2+2}\right) .
$$

Accordingly we have

$$
\|V\|_{m, 2, \Omega} \leqq C\|F\|_{m, 0, \Omega} .
$$

This implies $V \in \mathscr{H}_{m, 2}(\Omega)$. Hence $\partial V / \partial \nu \in \mathscr{H}_{m, I}(\Omega)$ and so $v^{\prime}=\partial V /\left.\partial \nu\right|_{\Gamma}$ is in $\mathscr{H}_{m}(\Gamma)$. As a matter of fact, we can obtain the inequality

$$
\langle v\rangle_{m} \leqq C \sum_{k=0}^{m}\left\|h^{2 k} v\right\|_{k}, \quad v \in C^{\infty}(\Gamma),
$$

applying (4.18) with $u=h^{2 k-2} v$. From this the inequality

$$
\left\langle v^{\prime}\right\rangle_{m} \leqq C\|V\|_{m, 2, \Omega}
$$

is easily derived. Accordingly, Theorem 4.2 (i) guarantees that the equation

$$
h(\alpha S-\gamma+\beta) w=h\left(f-\alpha v^{\prime}\right)
$$

admits a solution $w$ in $\mathscr{H}_{m}(\Gamma)$ if and only if $h\left(f-\alpha v^{\prime}\right)$ is orthogonal to $\tilde{N}_{m}$.

Let $w$ be a solution in $\mathscr{H}_{m}(\Gamma)$ of (4.23). It then follows from Propositions 1.1 and 1.2 that the distribution on $\Omega_{1}$,

$$
W=i^{-1} \sum_{j=0}^{1} \sum_{k=0}^{1-j} G A_{j+k+1} D_{m}^{j}\left(w_{k} \delta\right)\left(w_{0}=w, w_{1}=i S_{0} w\right)
$$

belongs to $\mathscr{H}_{m, 1 / 2}(\Omega)$, and satisfies $A W=0$ in $\Omega, W=w$ on $\Gamma$ and

$$
h\left(\alpha \frac{\partial W}{\partial \nu}+\gamma W+\beta W\right)=h\left(f-\alpha v^{\prime}\right)
$$

on $\Gamma$. In fact, we have

$$
\begin{aligned}
\eta^{k} W & =i^{-1} \sum_{j=0}^{1} \sum_{\ell=0}^{1-i}\left\{G A_{j+\ell+1} D_{n}^{j} \eta^{k}\left(w_{l} \delta\right)+\left[\eta^{k}, G A_{j+\ell+1} D_{n}^{j}\right]\left(w_{\ell} \delta\right)\right\} \\
& =i^{-1} \sum_{j=0}^{1} \sum_{\ell=0}^{1-j}\left\{G A_{j+\ell+1} D_{n}^{j}\left(h^{2 k} w_{\ell} \delta\right)+\sum_{i=0}^{k-1} P_{-(\ell+k+1-i)} \eta^{\imath}\left(w_{\ell} \delta\right)\right\},
\end{aligned}
$$

where $P_{-j}(i=\ell+2, \cdots, \ell+k+1)$ are pseudo-differential operators on $\Omega_{1}$ of order $-j$. Following [2, Section 2.1], we can obtain

$$
\left\|\eta^{k} W\right\|_{k+1 / 2, \Omega} \leqq C_{k} \sum_{i=0}^{k}\left(\left\|h^{2 i} w_{0}\right\|_{i}+\left\|h^{2 i} w_{1}\right\|_{i-1}\right)
$$


with a suitable constant $C_{k}$. Accordingly

$$
\|W\|_{m, 1 / 2, \Omega} \leqq C\langle w\rangle_{m},
$$

which immediately implies $W \in \mathscr{H}_{m, 1 / 2}(\Omega)$. Thus it follows that $U=V+$ $W$ is in $\mathscr{H}_{m, 1 / 2}(\Omega)$ and satisfies (4.20). Conversely, let $U \in \mathscr{H}_{m, 1 / 2}(\Omega)$ be a solution of (4.20). Then $W=U-V$ satisfies $A W=0$ in $\Omega$ and (4.25), and hence $w=\left.W\right|_{\Gamma}$ satisfies (4.23). Since $W \in \mathscr{H}_{m, 1 / 2}(\Omega) \subset H_{1 / 2}(\Omega)$, we have, by Proposition 1.1 (a), $w \in H_{0}(\Gamma)=\mathscr{H}_{0}(\Gamma)$. Therefore Theorem 4.2 (ii) guarantees $w \in \mathscr{H}_{m}(\Gamma)$, since right hand side of (4.23) is contained in $\mathscr{H}_{m}(\Gamma)$. Thus we could show that the problem (4.20) admits a solution in $\mathscr{H}_{m, 1 / 2}(\Omega)$ if and only if $h\left(f-\alpha v^{\prime}\right)$ is orthogonal to $\tilde{N}_{m}$. Now if $U$ is in $\mathscr{H}_{m, 1 / 2}(\Omega)$ and satisfies (4.20) with $F=f=0$, as we have seen above, $u=$ $\left.U\right|_{\Gamma}$ belongs to $\mathscr{H}_{m}(\Gamma)$ and satisfies $h(\alpha S-\gamma+\beta) u=0$. These complete the proof of (i).

(ii) Let $(F, f) \in \mathscr{H}_{\ell, 0}(\Omega) \times \mathscr{H}_{\ell}(\Gamma)$ and $U$ be a solution in $\mathscr{H}_{m, 1 / 2}(\Omega)$ of (4.20). Set $W=U-V$. Note that $V \in \mathscr{H}_{m, 2}(\Omega)$. Then $w=\left.W\right|_{\Gamma}$ satisfies (4.23) whose right hand side belongs to $\mathscr{H}_{\ell}(\Gamma)$. Therefore in virtue of Theorem 4.2 (ii), we have $w \in \mathscr{H}_{\ell}(\Gamma)$, which together with (4.26) proves $U \in \mathscr{H}_{m, 1 / 2}(\Omega)$.

Q.E.D.

As a corollary of Theorems 4.3 and 4.4, we can state

THeOREM 4.5. Let $\lambda_{0}$ be the number introduced in (4.16) and $\lambda$ be any real number such that $\lambda \geqq \lambda_{0}$. Then for every $(F, f) \in \mathscr{H}_{m, 0}(\Omega) \times \mathscr{H}_{m}(\Gamma)(m$ integer $\geqq 0$ ), we can find one and only one $U \in \mathscr{H}_{m, 1 / 2}(\Omega)$ satisfying

$$
\left\{\begin{array}{l}
(A+\lambda) U=F \quad \text { in } \\
h\left(\alpha \frac{\partial U}{\partial \nu}-\gamma U+\beta U\right) U=h f \quad \text { on } \Gamma .
\end{array}\right.
$$

Moreover the inequality

$$
\|U\|_{m, 1 / 2, \Omega} \leqq C_{m}\left(\|F\|_{m, 0, \Omega}+\langle f\rangle_{m}\right) .
$$

holds with a suitable constant $C_{m}>0$.

Proof. The first half of the theorem is obvious. We only prove (4.27). Let $V$ and $v$ be the same as in the proof of the preceding theorem and $w$ be a solution in $\mathscr{H}_{m}(\Gamma)$ of (4.23). Then we can write as $U=V+W$, where $W$ is defined by (4.24). Consequently, we have by (4.21), (4.22) and (4.26) 


$$
\begin{aligned}
\|U\|_{m, 1 / 2, \Omega} & \leqq\|V\|_{m, 1 / 2, \Omega}+\|W\|_{m, 1 / 2, \Omega} \leqq\|V\|_{m, 1 / 2, \Omega}+C_{1}\langle w\rangle_{m} \\
& \leqq C_{2}\left(\|V\|_{m, 2, \Omega}+\langle f\rangle_{m}\right) \leqq C_{3}\left(\|F\|_{m, 0, \Omega}+\langle f\rangle_{m}\right) .
\end{aligned}
$$

\section{Appendix}

Lemma A.1 (see Lemma 1 in [5]). Let $f(x)$ be in $C^{\infty}\left(R^{m}\right)$ and $P$ be a pseudo-differential operator on $R^{m}$ of order $t$. Then there exist pseudodifferential operators $P_{j}(j=1, \cdots, m)$ and $Q$ on $R^{m}$ of order $t-1$ and $t-2$, respectively, such that

$$
[f, P]=\sum_{j=1}^{m} \frac{\partial f}{\partial x_{j}} P_{j}+Q .
$$

Lemma A.2 (see Lemma A.1 in [3]). Let $f(x)$ be in $C_{0}^{\infty}\left(R^{m}\right)$ such that $f(x) \geqq 0$ in $R^{m}$. Then

$$
\left|\frac{\partial f}{\partial x_{j}}(x)\right|^{2} \leqq 2 K_{j} f(x), \quad x \in R^{m}(j=1, \cdots, m),
$$

where

$$
K_{j}=\sup _{x \in R^{m}}\left|\frac{\partial^{2} f}{\partial x_{j}^{2}}(x)\right| .
$$

Lemma A.3 (see Lemma 3 in [4]). Let $\Omega$ be a bounded domain in $R^{m}$ with $C^{\infty}$ boundary of dimension $m-1$ and let $q(x)$ be in $C^{\infty}(\bar{\Omega})$ such that $q(x)>0$ in $\Omega$ and $C$ dis $(x, \Gamma) \leqq q(x)$ in $\Omega_{d}=\{x \in \bar{\Omega}$; dis $(x, \Gamma)<d\}$ with suitable constant $C>0$ and $d>0$. Then for any $\delta>0$ there exists $a$ constant $C_{\delta}>0$ such that

$$
\|U\|_{0, \Omega}^{2} \leqq \delta\|p \partial U\|_{0, \Omega}^{2}+C_{\delta}\|p U\|_{0}^{2}, \quad U \in C^{\infty}(\bar{\Omega}),
$$

where $p=\sqrt{q}$.

\section{REFERENCES}

[1] Ju. V. Egorov and V. A. Kondrat'ev, The oblique derivative problems, Mat. Sbornik, 78 (1969), 148-176. = Math. USSR Sbornik, 7 (1969), 139-169.

[2] L. Hörmander, Pseudo-differential operators and non-elliptic boundary problem Ann. of Math., 83 (1966), 129-209.

[3] Y. Kato, Mixed-type boundary condition for second order elliptic differential equations, J. Math. Soc. Japan, 26 (1974), 405-432.

[4. ] - On a class of non-elliptic boundary problems, Nagoya Math. J., 54 (1974), 7-20.

[5] - Another approach to a non-elliptic boundary problem, Nagoya Math. J., 66 (1977), 13-22. 
[6] M. B. Maljutov, On the Poincaré boundary value problem, Trans. Moscow Math. Soc., 20 (1969), 173-204.

[7] H. Soga, Boundary value problems with oblique derivative Publ. RIMS, Kyoto Univ., 10 (1975), 619-668.

[8] B. Winzell, Solutions of second order elliptic partial differential equations with prescribed directional derivative on the boundary, Dissertation.

[ 9 ] K. Yosida, Functional analysis, Springer-Verlag (1965).

Nagoya University 